\title{
Aeroelastic Tailoring Study of an N+2 Low-boom Supersonic Commercial Transport Aircraft
}

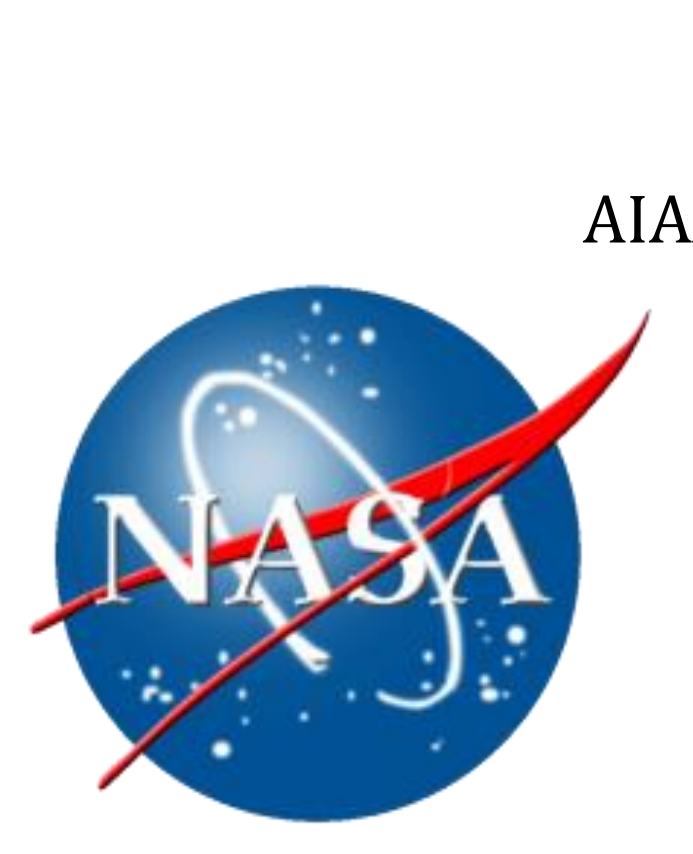

\author{
Prepared For: \\ AIAA Aviation 2015
}

AIAA/ISSMO Multidisciplinary Analysis and Optimization Conference

June 22-26, Dallas, Texas

\author{
Prepared By: \\ Chan-gi Pak, Ph.D.
}

Structural Dynamics Group, Aerostructures Branch (Code RS)

NASA Armstrong Flight Research Center 
Supersonic Commercial Transport Aircraft Design

- Game Change Approach in Aircraft Design

- Multidisciplinary Design Optimization tool

- Multidisciplinary Analysis of the Baseline Configuration

* Structural and Aerodynamic Models

* Modal Analyses

* Flutter Analyses

* Trim Analyses

* Landing and Ground Control Loads

* Buckling and Strength Analyses

$\square$ First Optimization Run

口 Second Optimization Run

口 Third Optimization Run

C Conclusions

口 Future Studies 


\section{(3) Supersonic Commercial Transport Aircraft Design}

( Major Issues

* Safety

$>$ Light weight airframe can cause strength, buckling, aeroelastic, and aeroservoelastic problems.

* Sonic boom

$>$ Supersonic flight of "commercial transport" aircraft allowed only over the ocean.

$>$ Perceived Loudness in decibels

$\checkmark$ NASA's N+2 goal: 85 PLdB

$\checkmark$ Concorde: 104 PLdB

$\checkmark$ High Speed Civil Transport (HSCT): 99 PLdB

* Fuel efficiency

$>$ Light weight airframe

$>$ Reduced drag

Developing N+2 Low-boom Supersonic Commercial Transport (LSCT) aircraft

* Boeing

* Lockheed Martin: 79 PLdB

* Gulf Stream

* Aerion with "Airbus"

Boeing

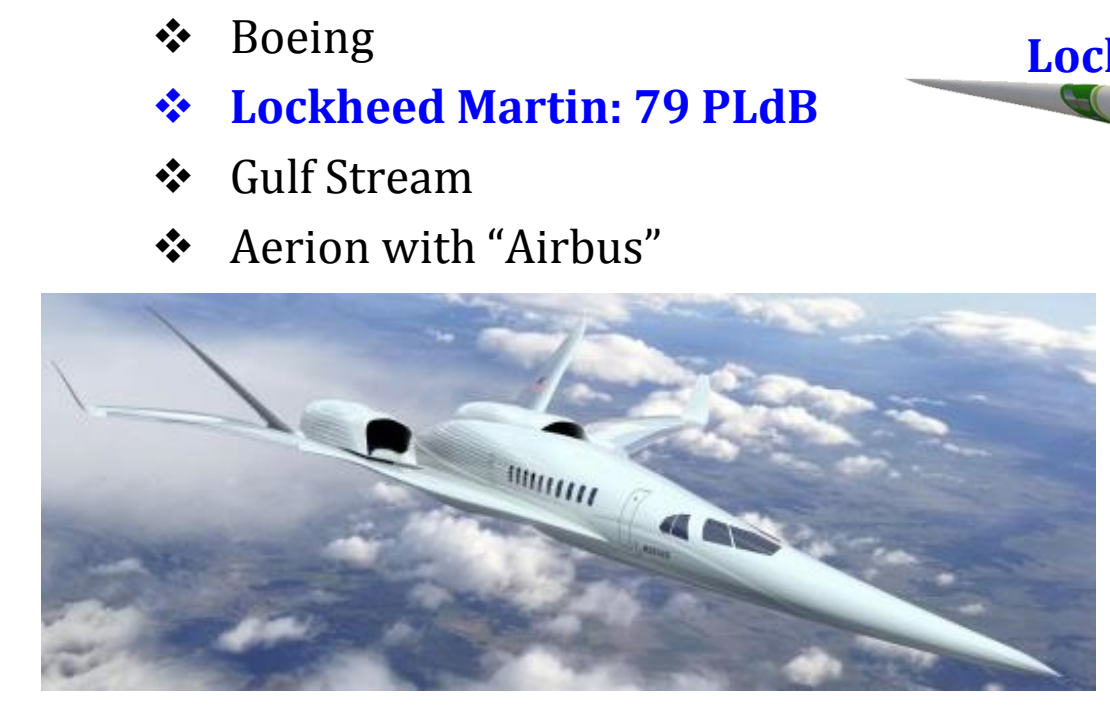

Lockheed Martin

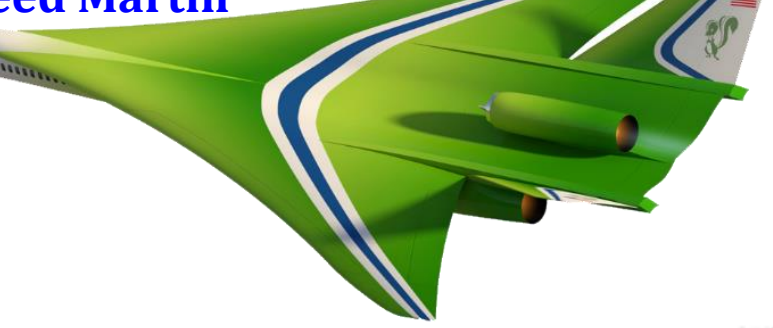

Concorde

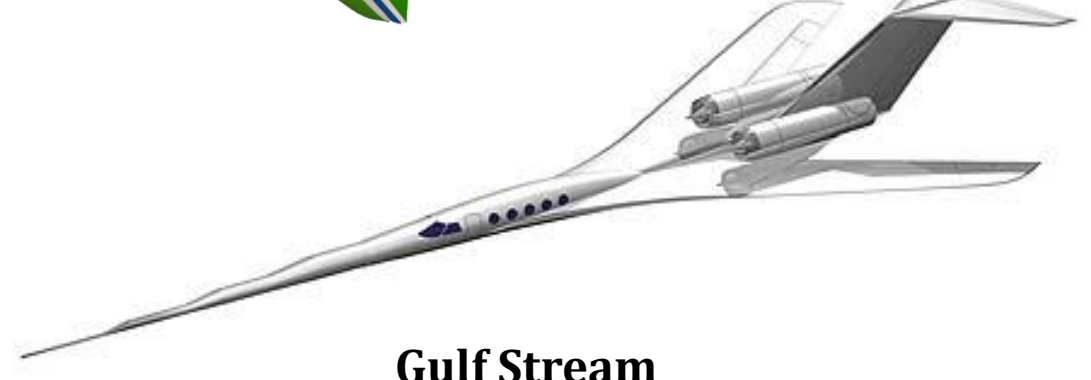

Gulf Stream

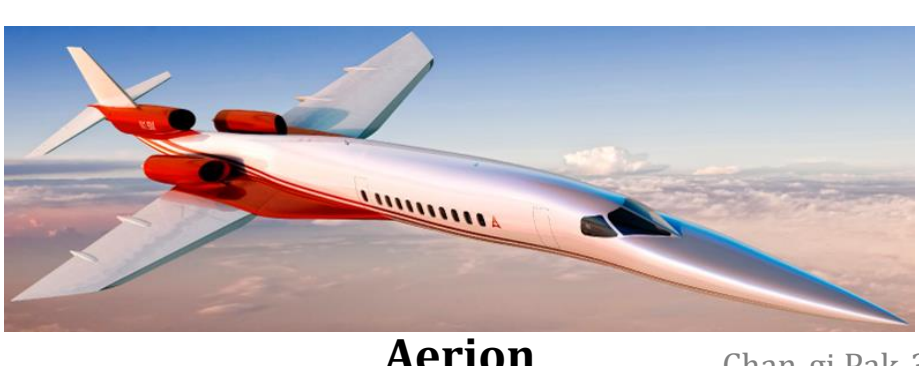

Aerion 


\section{Game Change Approach in Aircraft Design}

\section{Problem Statement}

Design innovations are needed to further down the weight of an aircraft which current design technologies can take care of.

\section{Long Term Objective}

- Use aeroelastic tailoring theory and active flexible motion control technique to satisfy the overall strain, aeroelastic, and aeroservoelastic instability requirements within given flight envelopes

Use curvilinear sparib concept as well as composite ply angles for aeroelastic tailoring

\section{Approach}

- Simultaneously update structural as well as control design variables during early design phase

* Perform topology optimization with curvilinear sparibs

* Use aeroelastic tailoring up to $\mathrm{V}_{\mathrm{L}}$

* Use aeroservoelastic tailoring between $\mathrm{V}_{\mathrm{L}}$ and $1.15 \mathrm{~V}_{\mathrm{L}}$

\section{Current Study}

O Optimize baseline aircraft model

* Use Lockheed Martin's configuration

* Use aeroelastic tailoring up to $1.15 \mathrm{~V}_{\mathrm{L}}$
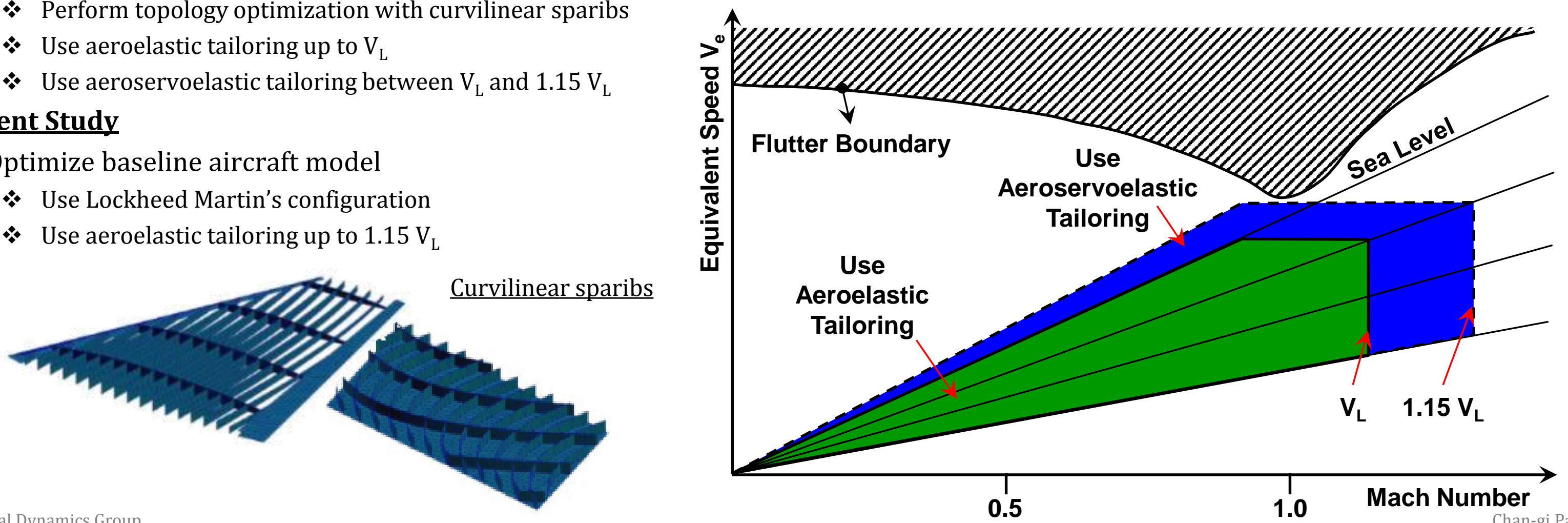


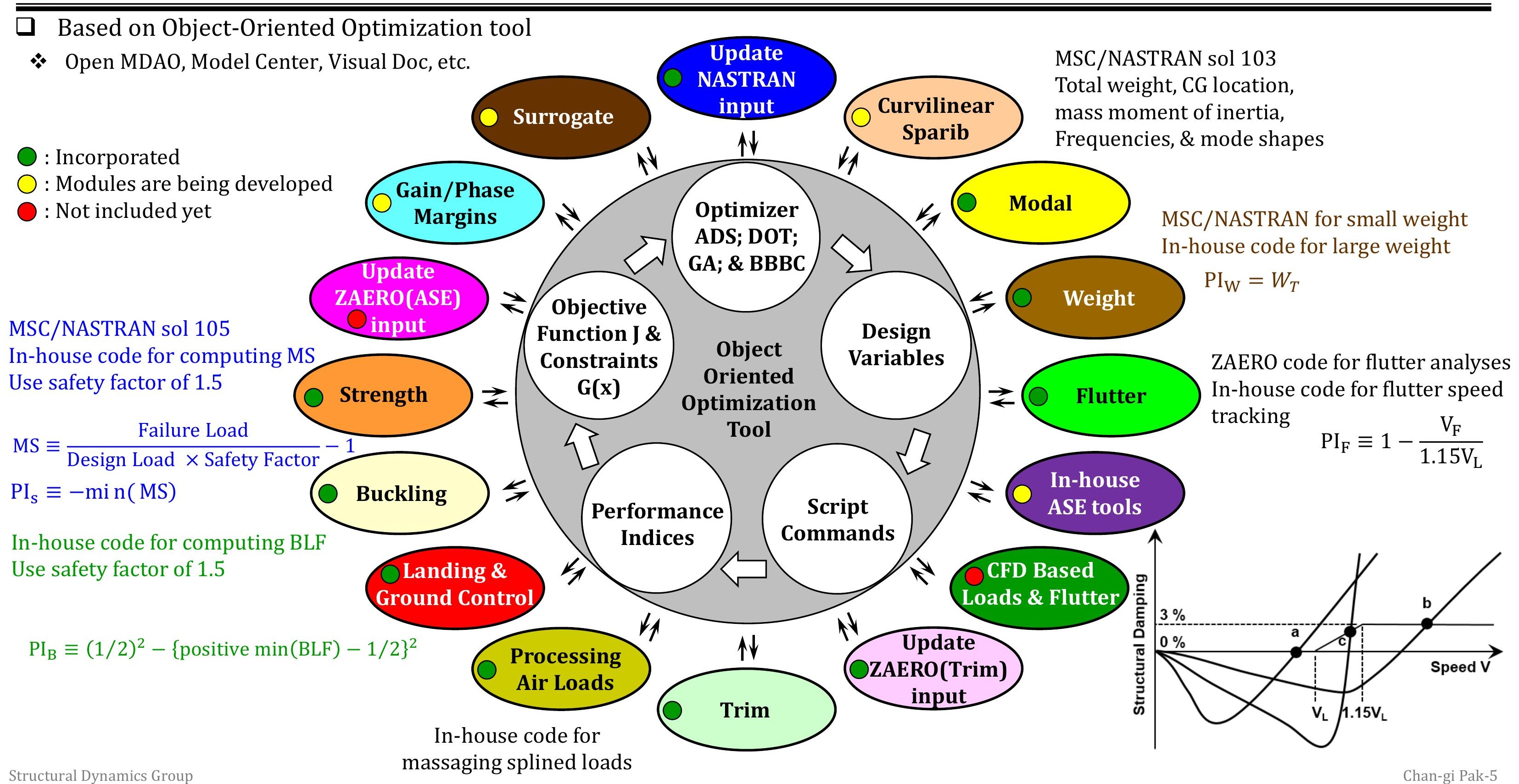


Multidisciplinary Analysis of the Baseline Configuration 


\section{Structural and Aerodynamic Models}

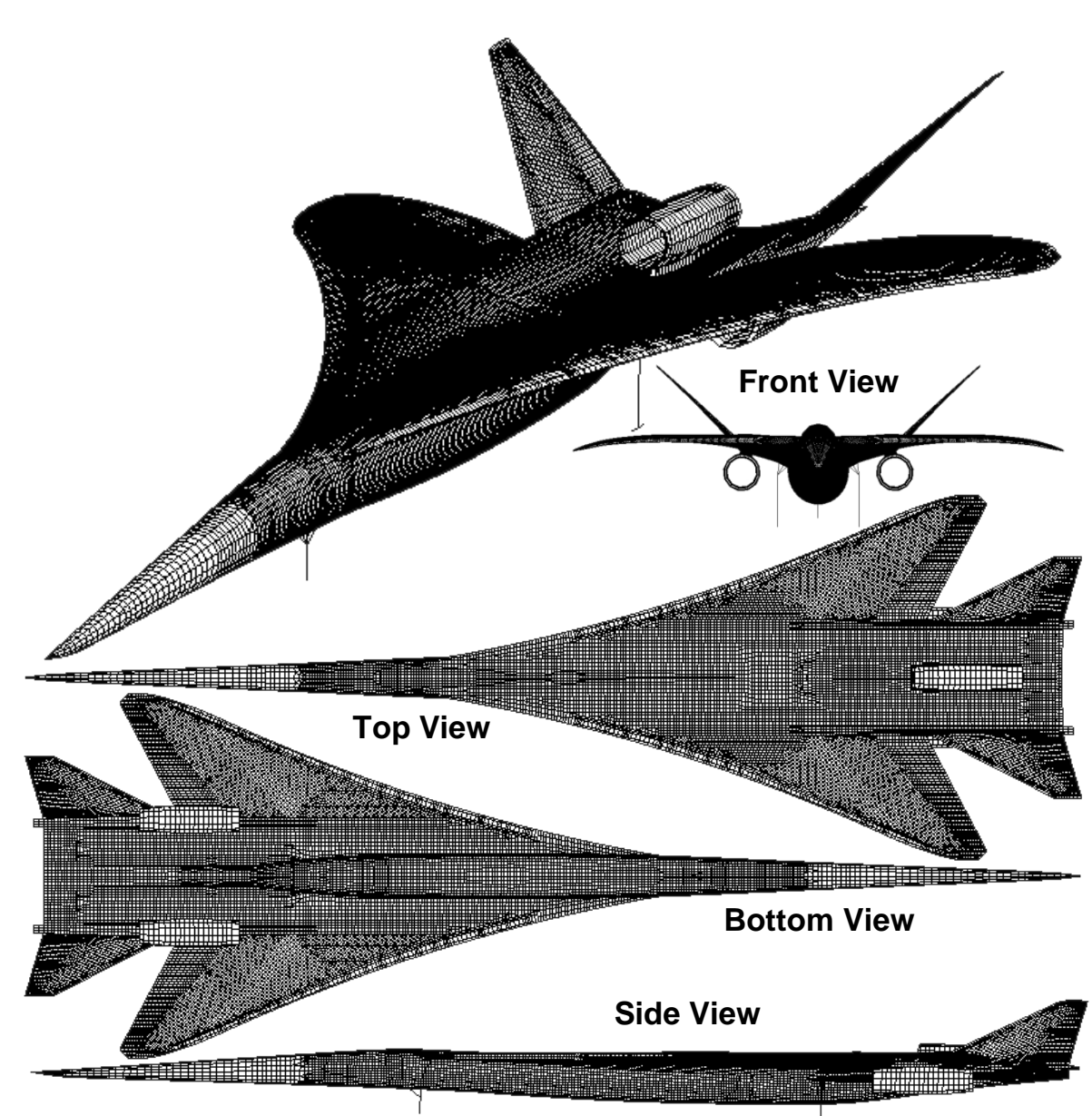

$\square$ MSC/NASTRAN structural model

* Total number of grids: 55,635

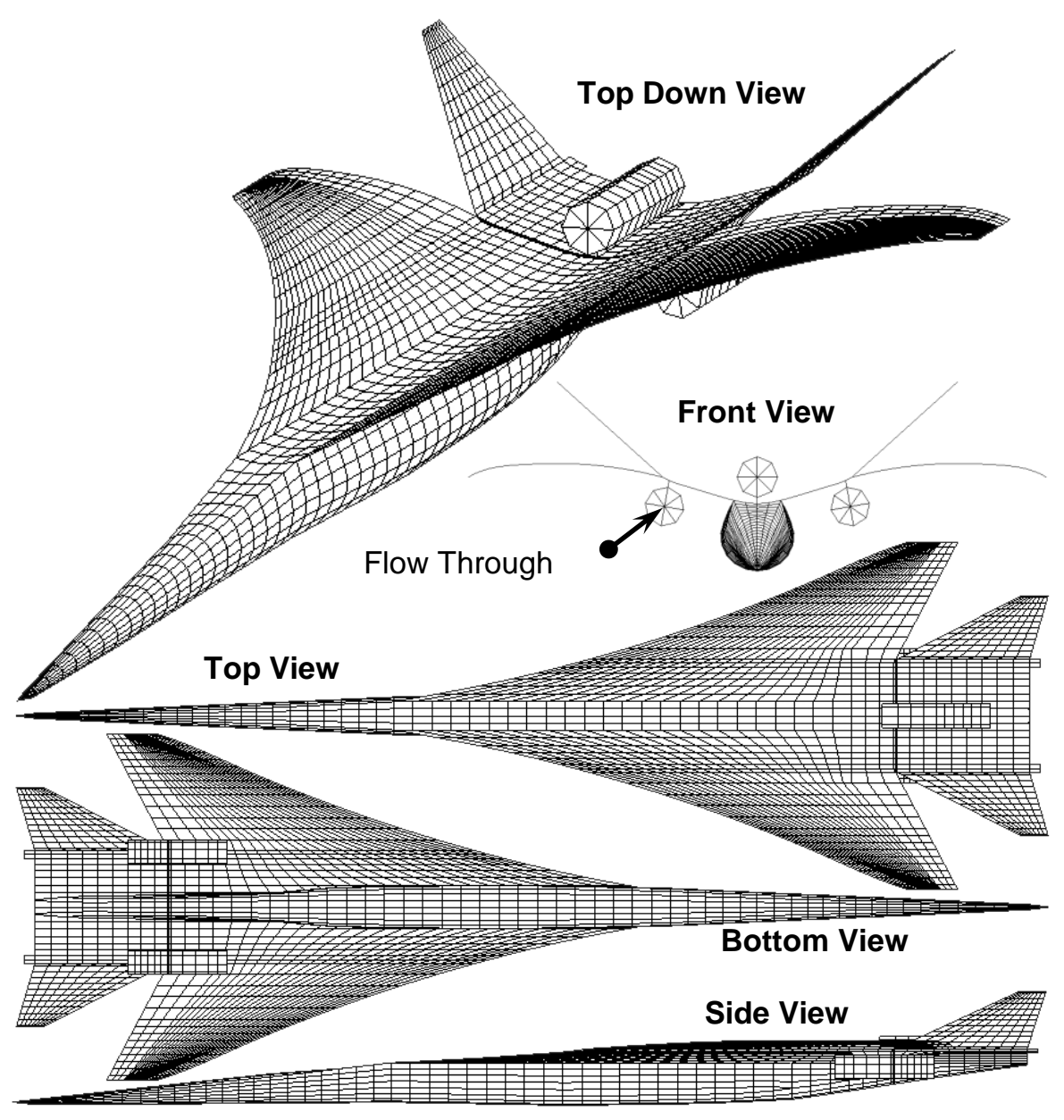

$\square$ ZAERO unsteady aerodynamic model

* 5,060 surface elements

* Six Mach numbers: 0.66, 0.89, 1.41, 1.80, 2.00, and 2.30

* Sixteen reduced frequencies: 0., 0.005, 0.01, 0.05, 0.10, 0.15, 0.20, $0.25,0.30,0.35,0.40,0.45,0.50,0.65,0.80,1.0$ 


\section{Modal Analyses of the Baseline Configuration}

ㅁased on six configurations

* Gear up

$>$ DTOW(Design Take Off Weight), FFEP(Full Fuel Empty Payload), M2W(Mach 2 Weight), \& ZFW(Zero Fuel Weight)

$\checkmark$ DTOW=FFFP (Full Fuel Full Payload)

$\checkmark$ ZFW=EFFP (Empty Fuel Full Payload)

- Gear down

$>$ DTOW(or FFFP) and DLW(Design Landing Weight)

\begin{tabular}{|c|c|c|c|c|c|c|c|}
\hline \multirow{3}{*}{$\begin{array}{c}\text { Mode } \\
\text { Number }\end{array}$} & \multicolumn{6}{|c|}{ Natural Frequency $(\mathrm{Hz})$} & \multirow{3}{*}{ Notes } \\
\hline & \multicolumn{4}{|c|}{ Gear-up } & \multicolumn{2}{|c|}{ Gear-down } & \\
\hline & DTOW & FFEP & M2W & ZFW & DTOW & DLW & \\
\hline 7 & 2.049 & 2.055 & 2.071 & 2.266 & 2.048 & 2.158 & Aft fuselage torsion \\
\hline 8 & 2.235 & 2.262 & 2.277 & 2.554 & 2.238 & 2.424 & First symmetric fuselage bending \\
\hline 9 & 2.498 & 2.509 & 2.539 & 2.993 & 2.503 & 2.714 & First symmetric wing bending \\
\hline 10 & 2.754 & 2.769 & 2.935 & 3.415 & 2.752 & 3.265 & First anti-symmetric wing bending \\
\hline 11 & 3.060 & 3.069 & 3.115 & 3.731 & 3.057 & 3.403 & Symmetric tail bending \\
\hline 12 & 3.562 & 3.608 & 3.689 & 4.044 & 3.574 & 3.945 & Forward fuselage lateral bending \\
\hline 13 & 4.440 & 4.449 & 4.511 & 4.790 & 4.429 & 4.602 & First anti-symmetric tail bending \\
\hline 14 & 4.456 & 4.537 & 4.555 & 5.532 & 4.437 & 5.142 & Second symmetric wing bending \\
\hline 15 & 4.818 & 4.842 & 5.146 & 5.832 & 4.809 & 5.542 & Second anti-symmetric wing bending \\
\hline 16 & 5.449 & 5.465 & 5.550 & 6.158 & 5.444 & 5.994 & Symmetric aft inner wing bending \\
\hline
\end{tabular}




\section{Flexible Mode Shapes (gear up: DTOW)}

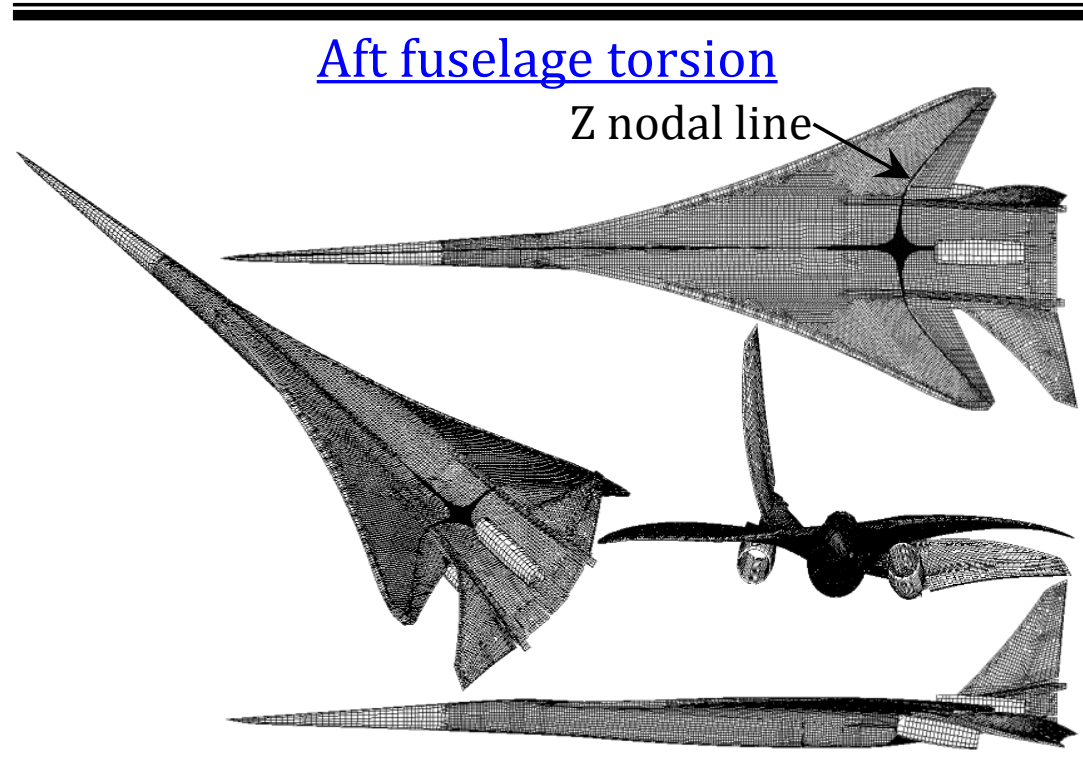

$1^{\text {st }}$ Mode: $2.049 \mathrm{~Hz}$

First anti-symmetric wing bending

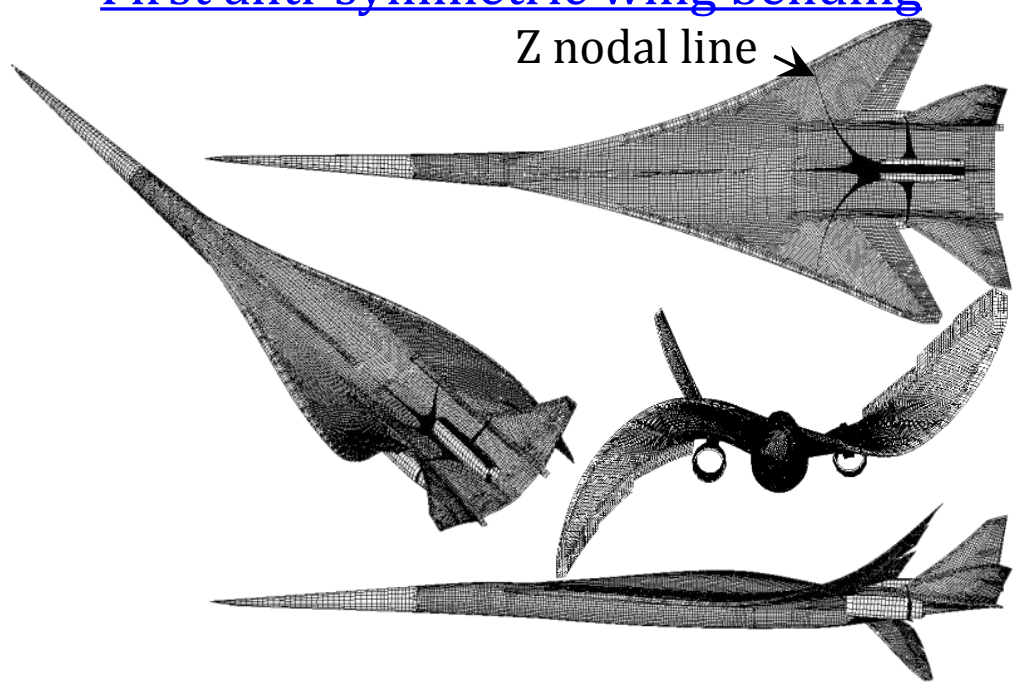

$4^{\text {th }}$ Mode: $2.754 \mathrm{~Hz}$
First symmetric fuselage bending

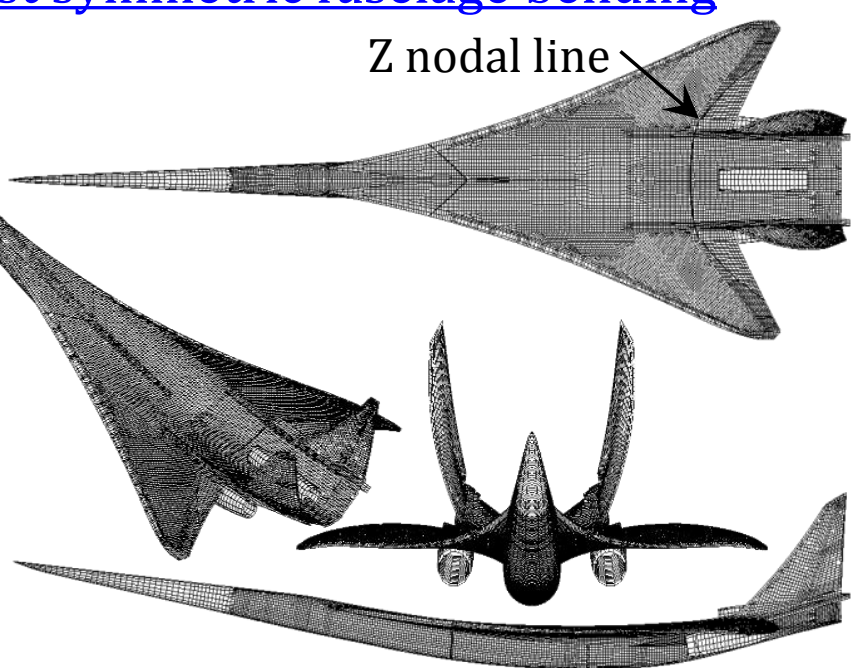

$2^{\text {nd }}$ Mode: $2.235 \mathrm{~Hz}$

Symmetric tail bending

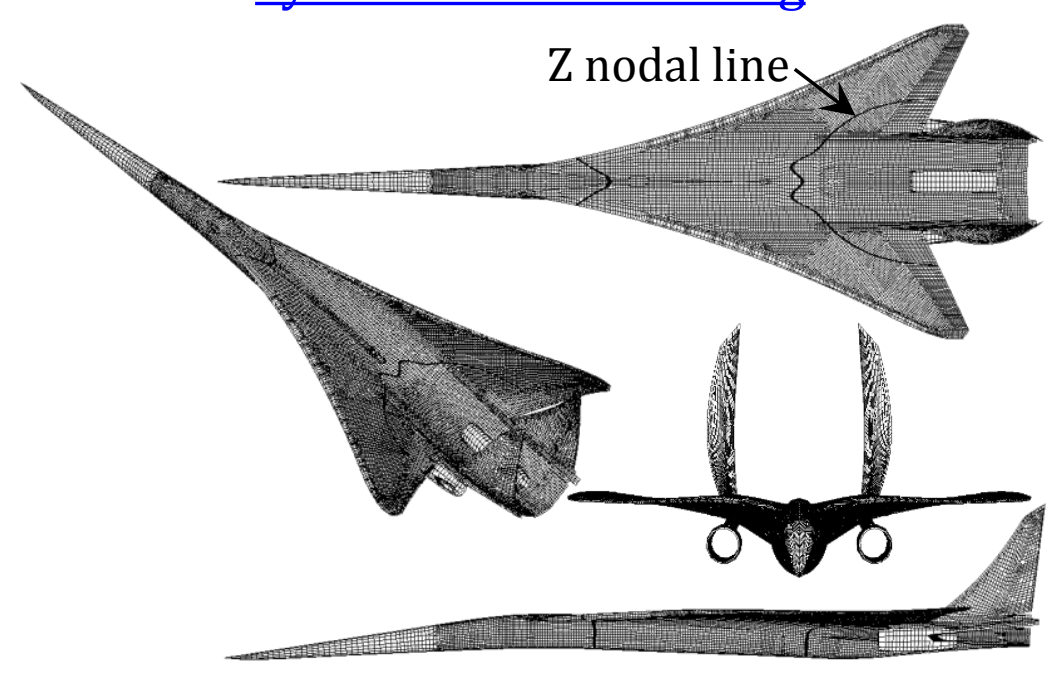

$5^{\text {th }}$ Mode: $3.060 \mathrm{~Hz}$
First symmetric wing bending

Z nodal line $>$

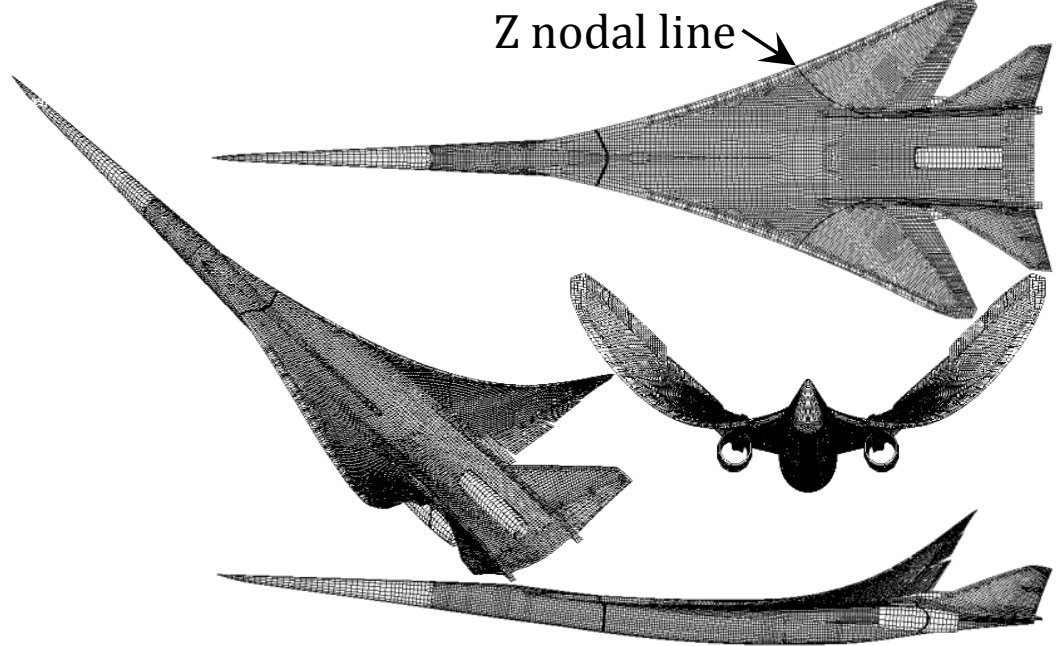

$3^{\text {rd }}$ Mode: $2.498 \mathrm{~Hz}$

Forward fuselage lateral bending

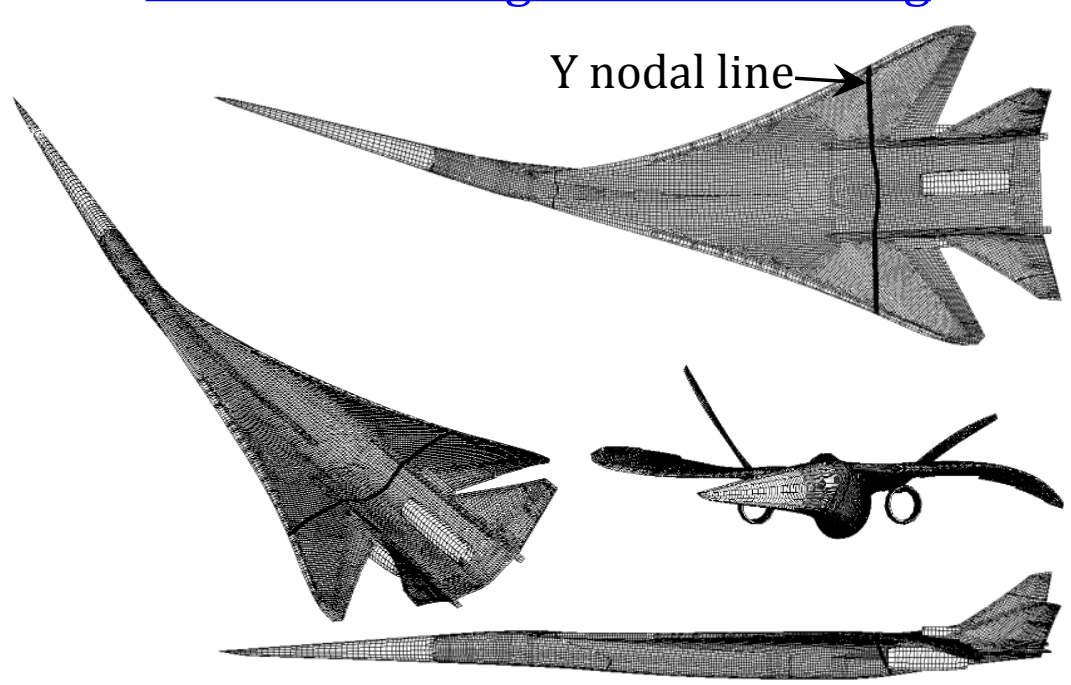

$6^{\text {th }}$ Mode: $3.562 \mathrm{~Hz}$ 


\section{Flutter Analyses of the Baseline Configuration}

- Based on four structural configurations at six Mach numbers * Gear up

$>$ DTOW, FFEP, EFEP, \& ZFW

$>$ Mach numbers of $0.66,0.89,1.41,1.80,2.00, \& 2.30$

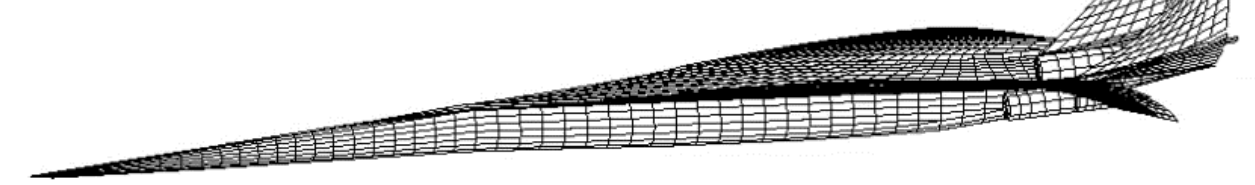

O: Empty Fuel Empty Payload (EFEP) $\triangle$ : Full Fuel Empty Payload (FFEP)

$\diamond:$ Zero Fuel Weight

(ZLW)

$\square$ : Design Take-Off Weight (DTOW)

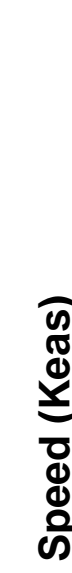
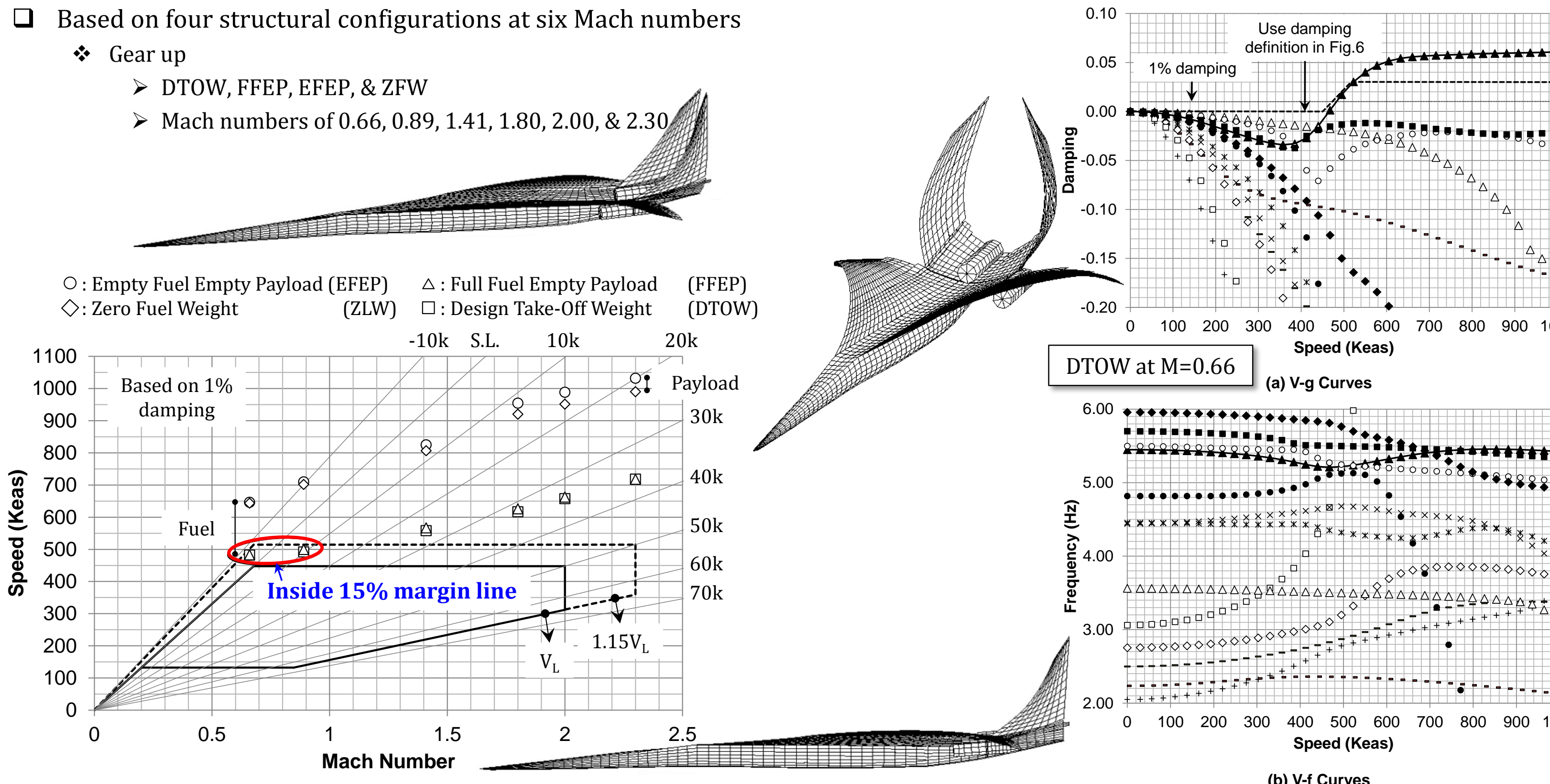

을 -0.05

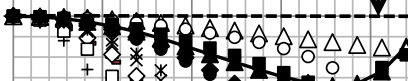

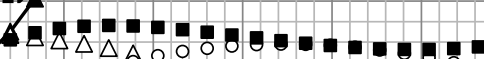
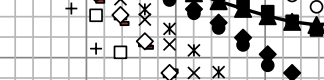

$\because 0$

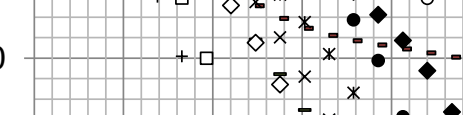

$-0.20$

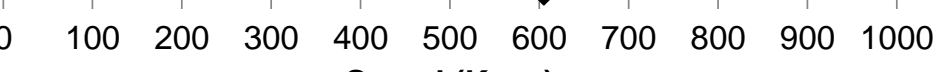

DTOW at $\mathrm{M}=0.66$

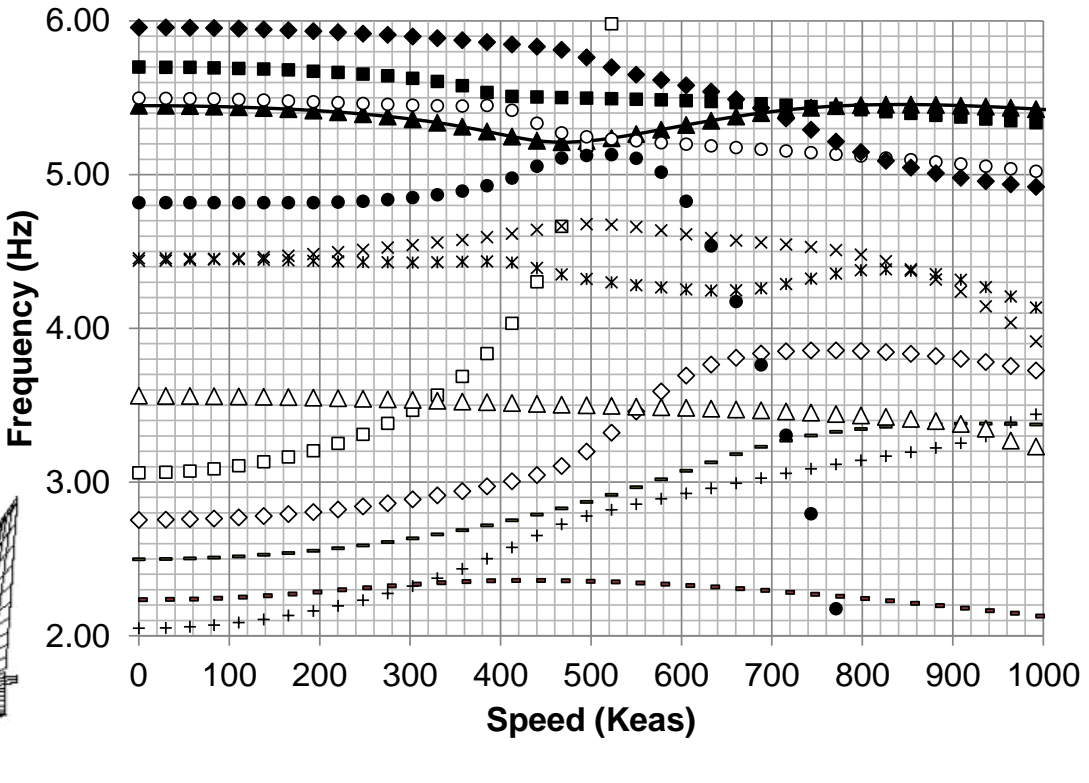

(b) V-f Curves 


\section{Trim Analyses of the Baseline Configuration}

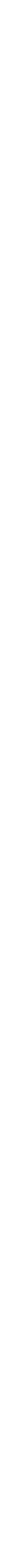




\section{Trim Analyses of the Baseline Configuration (continue)}

口 Trim results

- In general trim angles are larger than NASTRAN results.
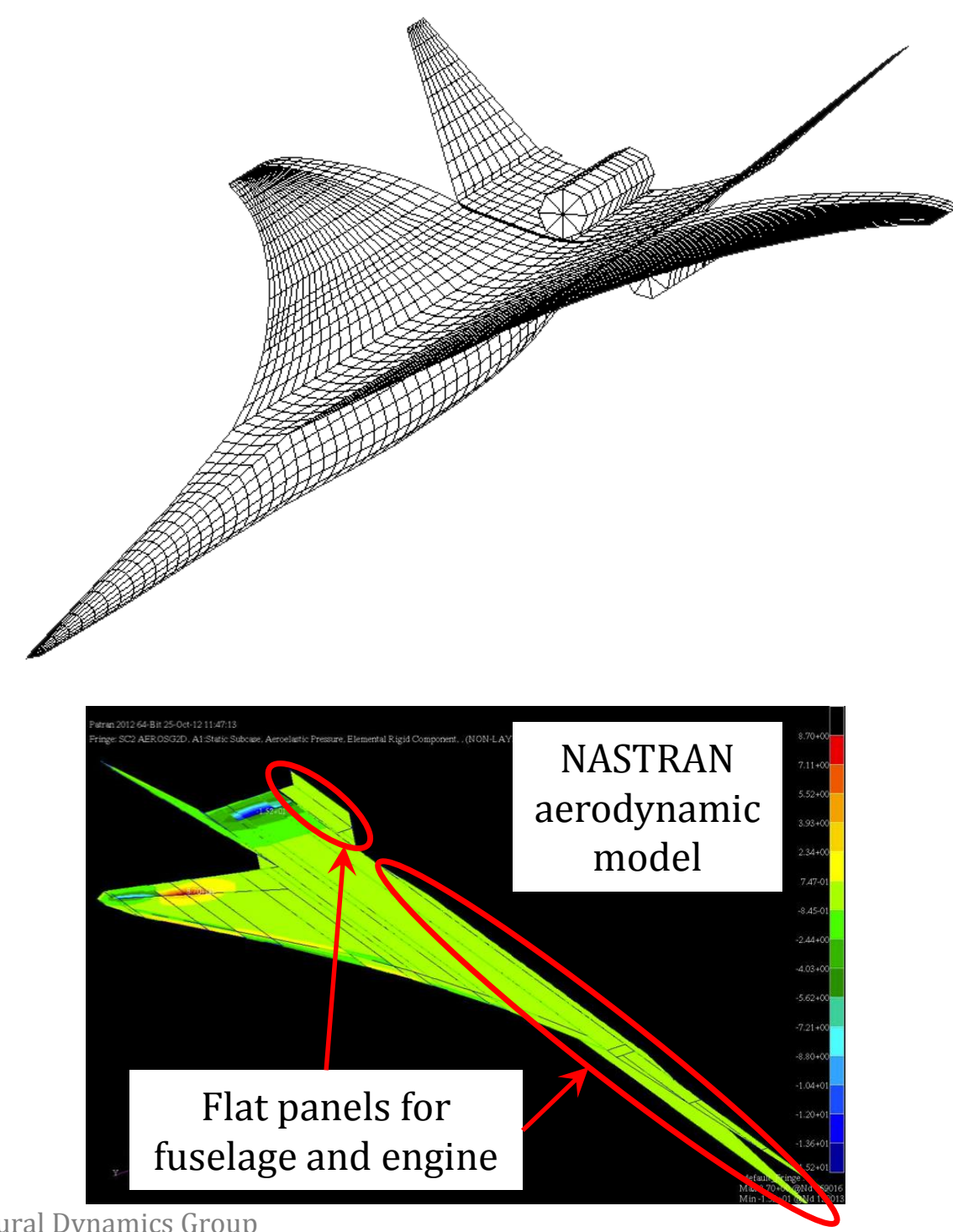

\begin{tabular}{|c|c|c|c|c|c|c|c|c|c|}
\hline Load Case & 100 & 200 & 300 & 400 & 500 & 600 & 700 & 800 & 900 \\
\hline Trim Analysis & \multicolumn{9}{|c|}{ Symmetric } \\
\hline $\mathrm{Nx}(\mathrm{G})$ & -0.007 & -0.003 & -0.005 & 0.001 & 0.002 & -0.004 & -0.016 & -0.006 & 0.002 \\
\hline $\mathrm{Nz}(\mathrm{G})$ & 2.5 & -1.0 & 2.5 & 2.5 & -1.0 & 2.5 & 2.5 & -1.0 & 2.5 \\
\hline $\operatorname{Pdot}\left(\mathrm{rad} / \mathrm{s}^{2} / \mathrm{g}\right)$ & None & None & None & None & None & None & None & None & None \\
\hline Qdot $\left(\mathrm{rad} / \mathrm{s}^{2} / \mathrm{g}\right)$ & 0.0 & 0.0 & 0.0 & 0.0 & 0.0 & 0.0 & 0.0 & 0.0 & $\overline{0.0}$ \\
\hline $\mathrm{Pb} / 2 \mathrm{~V}(\mathrm{rad})$ & None & None & None & None & None & None & None & None & None \\
\hline $\mathrm{Qc} / 2 \mathrm{~V}(\mathrm{rad})$ & 0.0 & 0.0 & 0.0 & 0.0 & 0.0 & 0.0 & 0.0 & 0.0 & 0.0 \\
\hline$\alpha\left(^{\circ}\right)$ & 7.75 & -2.50 & 14.37 & 8.32 & -2.81 & 16.90 & 4.10 & -1.04 & 5.07 \\
\hline Body Flap $\left(^{\circ}\right)$ & 2.01 & -6.07 & 6.12 & -5.25 & 5.42 & -25.68 & -8.01 & -1.59 & $-12.9^{\prime}$ \\
\hline Trailing-Edge Flap $\left(^{\circ}\right)$ & & & & -5.25 & & -25.68 & & & $-12.9^{\prime}$ \\
\hline Aileron \#1 $\left(^{\circ}\right)$ & & & & & & -25.68 & & & \\
\hline Aileron \#2 $\left(^{\circ}\right)$ & & & & & & -25.68 & & & \\
\hline Mach Number & 0.66 & 0.66 & 0.48 & 2.00 & 2.00 & 1.41 & 0.66 & 0.66 & 2.00 \\
\hline Altitude (ft) & SL & SL & SL & 49770 & 49770 & 49770 & SL & SL & 4977 \\
\hline Weight Configuration & DTOW & DTOW & DTOW & $\mathrm{M} 2 \mathrm{~W}$ & $\mathrm{M} 2 \mathrm{~W}$ & DTOW & ZFW & ZFW & ZFW \\
\hline Gear Configuration & Up & Up & Up & $\mathrm{Up}$ & $\mathrm{Up}$ & Up & Up & Up & Up \\
\hline Load Case & 1000 & 1100 & 1200 & 1300 & 1400 & 1500 & 1600 & 1700 & $\overline{1800}$ \\
\hline Trim Analysis & Sym. & \multicolumn{4}{|c|}{ Asymmetric (sym. + Anti-sym.) } & \multicolumn{4}{|c|}{ Symmetric } \\
\hline $\mathrm{Nx}(\mathrm{G})$ & 0.004 & -0.002 & -0.002 & -0.004 & -0.004 & -0.002 & 0.002 & -0.022 & -0.00 \\
\hline $\mathrm{Nz}(\mathrm{G})$ & -1.0 & 0.0 & 0.0 & 1.67 & 1.67 & 1.0 & 1.0 & 2.7 & 1.0 \\
\hline $\operatorname{Pdot}\left(\mathrm{rad} / \mathrm{s}^{2} / \mathrm{g}\right)$ & None & 0.0 & 0.0014 & 0.0 & 0.0014 & None & None & None & None \\
\hline Qdot $\left(\mathrm{rad} / \mathrm{s}^{2} / \mathrm{g}\right)$ & 0.0 & 0.0 & 0.0 & 0.0 & 0.0 & 0.0 & 0.0 & 0.0 & 0.0 \\
\hline $\mathrm{Pb} / 2 \mathrm{~V}(\mathrm{rad})$ & None & 0.0410 & 0.0 & 0.0410 & 0.0 & None & None & None & None \\
\hline $\mathrm{Qc} / 2 \mathrm{~V}(\mathrm{rad})$ & 0.0 & 0.0 & 0.0 & 0.0 & 0.0 & 0.0 & 0.0 & 0.0 & 0.0 \\
\hline$\alpha\left(^{\circ}\right)$ & -1.44 & 0.40 & 0.40 & 9.74 & 9.74 & 13.91 & 6.02 & 4.95 & 9.07 \\
\hline Body Flap $\left(^{\circ}\right)$ & 11.89 & -2.86 & -2.86 & 3.14 & 3.14 & 8.00 & -9.87 & -13.20 & 22.52 \\
\hline Trailing-Edge Flap $\left(^{\circ}\right)$ & & & & & & & -9.87 & & \\
\hline Aileron \#1 $\left(^{\circ}\right)$ & & 19.07 & 48.63 & 19.07 & 48.63 & & & & \\
\hline Aileron \#2 $\left(^{\circ}\right)$ & & 19.07 & 48.63 & 19.07 & 48.63 & & & & \\
\hline Mach Number & 2.00 & 0.48 & 0.48 & 0.48 & 0.48 & 0.3092 & 1.80 & 0.89 & $\overline{0.309}$ \\
\hline Altitude (ft) & 49770 & SL & SL & SL & SL & SL & 55000 & 20000 & $\mathrm{SL}$ \\
\hline Weight Configura & ZFW & DTOW & DTOW & DTOW & DTOW & DTOW & DTOW & ZFW & DLW \\
\hline Gear Configuration & Up & Up & Up & Up & Up & Down & Up & Up & Dow! \\
\hline
\end{tabular}


Landing and Ground Control Loads of the Baseline Configuration

\section{口 Landing loads}

Ground control loads

- Emergency landing loads (applied to three engine structures)

* 9G forward loading; 1.5G rearward loading; 3G sideway loading; \& 6G downward loading

\begin{tabular}{|c|c|c|c|c|c|}
\hline Load Cases & Case Number & Load & Right-MLG & Left-MLG & NLG \\
\hline \multirow{3}{*}{$\begin{array}{l}\text { Level + } \\
\text { Trim load }\end{array}$} & \multirow{3}{*}{$\begin{array}{l}3001 \text { (DTOW) } \\
\& 4001 \text { (DLW) }\end{array}$} & $\mathrm{FX}$ & $0.25 \mathrm{FM}_{L v}$ & $0.25 \mathrm{FM}_{L v}$ & $0.25 \mathrm{FN}_{L v}$ \\
\hline & & $\mathrm{FY}$ & 0.0 & 0.0 & 0.0 \\
\hline & & FZ & $\mathrm{FM}_{L v}=\mathrm{f}_{L M G} \mathrm{~W}_{T}$ & $\mathrm{FM}_{L v}$ & $\mathrm{FN}_{L v}=\mathrm{f}_{L N G} \mathrm{~W}_{T}$ \\
\hline \multirow{3}{*}{$\begin{array}{l}\text { Spin up + } \\
\text { Trim load }\end{array}$} & \multirow{3}{*}{$\begin{array}{l}3002 \text { (DTOW) } \\
\& 4002 \text { (DLW) }\end{array}$} & FX & $(0.8 \times 0.8) \mathrm{FM}_{L v}$ & $(0.8 \times 0.8) \mathrm{FM}_{L v}$ & $(0.8 \times 0.8) \mathrm{FN}_{L v}$ \\
\hline & & FY & 0.0 & 0.0 & 0.0 \\
\hline & & FZ & $0.8 \mathrm{FM}_{L v}$ & $0.8 \mathrm{FM}_{L v}$ & $0.8 \mathrm{FN}_{L v}$ \\
\hline \multirow{3}{*}{$\begin{array}{l}\text { Spring back } \\
\text { + Trim load }\end{array}$} & \multirow{3}{*}{$\begin{array}{l}3003 \text { (DTOW) } \\
\& 4003 \text { (DLW) }\end{array}$} & $\mathrm{FX}$ & $-(0.8 \times 0.8) \mathrm{FM}_{L v}$ & $-(0.8 \times 0.8) \mathrm{FM}_{L v}$ & $-(0.8 \times 0.8) \mathrm{FN}_{L v}$ \\
\hline & & FY & 0.0 & 0.0 & 0.0 \\
\hline & & $\mathrm{FZ}$ & $0.8 \mathrm{FM}_{L v}$ & $0.8 \mathrm{FM}_{L v}$ & $0.8 \mathrm{FN}_{L v}$ \\
\hline \multirow{3}{*}{$\begin{array}{c}\text { Lateral } \\
\text { drift }+ \\
\text { Trim load } \\
\end{array}$} & \multirow{3}{*}{$\begin{array}{l}3004 \text { (DTOW) } \\
\& 4004 \text { (DLW) }\end{array}$} & $\mathrm{FX}$ & $(0.4 \times 0.75) \mathrm{FM}_{L v}$ & $(0.4 \times 0.75) \mathrm{FM}_{L v}$ & $0.4 \mathrm{FN}_{L v}$ \\
\hline & & FY & $(0.25 \times 0.75) \mathrm{FM}_{L v}$ & $(0.25 \times 0.75) \mathrm{FM}_{L v}$ & $0.25 \mathrm{FN}_{L v}$ \\
\hline & & $\mathrm{FZ}$ & $0.75 \mathrm{FM}_{L v}$ & $0.75 \mathrm{FM}_{L v}$ & $\mathrm{FN}_{L v}$ \\
\hline \multirow{3}{*}{$\begin{array}{c}\text { Right one } \\
\text { gear + Trim } \\
\text { load } \\
\end{array}$} & \multirow{3}{*}{$\begin{array}{l}3005 \text { (DTOW) } \\
\& 4005 \text { (DLW) }\end{array}$} & $\mathrm{FX}$ & $0.25 \mathrm{FM}_{L v}$ & 0.0 & 0.0 \\
\hline & & FY & 0.0 & 0.0 & 0.0 \\
\hline & & FZ & $\mathrm{FM}_{L v}$ & 0.0 & 0.0 \\
\hline \multirow{3}{*}{$\begin{array}{c}\text { Left one } \\
\text { gear + Trim } \\
\text { load } \\
\end{array}$} & \multirow{3}{*}{$\begin{array}{l}3006 \text { (DTOW) } \\
\& 4006 \text { (DLW) }\end{array}$} & FX & 0.0 & $0.25 \mathrm{FM}_{L v}$ & 0.0 \\
\hline & & FY & 0.0 & 0.0 & 0.0 \\
\hline & & $\mathrm{FZ}$ & 0.0 & $\mathrm{FM}_{L v}$ & 0.0 \\
\hline \multirow{3}{*}{$\begin{array}{c}\text { Side load } \\
\text { RtoL }+ \\
\text { Trim load } \\
\end{array}$} & \multirow{3}{*}{$\begin{array}{l}3007 \text { (DTOW) } \\
\& 4007 \text { (DLW) }\end{array}$} & FX & 0.0 & 0.0 & 0.0 \\
\hline & & $\mathrm{FY}$ & $(0.8 \times 0.5) \mathrm{FM}_{L v}$ & $(0.6 \times 0.5) \mathrm{FM}_{L v}$ & 0.0 \\
\hline & & FZ & $0.5 \mathrm{FM}_{L v}$ & $0.5 \mathrm{FM}_{L v}$ & 0.0 \\
\hline \multirow{3}{*}{$\begin{array}{c}\text { Side load } \\
\text { LtoR }+ \\
\text { Trim load }\end{array}$} & \multirow{3}{*}{$\begin{array}{l}3008 \text { (DTOW) } \\
\& 4008 \text { (DLW) }\end{array}$} & FX & 0.0 & 0.0 & 0.0 \\
\hline & & $\mathrm{FY}$ & $-(0.6 \times 0.5) \mathrm{FM}_{L v}$ & $-(0.8 \times 0.5) \mathrm{FM}_{L v}$ & 0.0 \\
\hline & & $\mathrm{FZ}$ & $0.5 \mathrm{FM}_{L v}$ & $0.5 \mathrm{FM}_{L v}$ & 0.0 \\
\hline \multicolumn{2}{|l|}{ DTOW } & \multicolumn{4}{|c|}{$\mathrm{f}_{L M G}=0.36 ; \mathrm{f}_{L N G}=0.0639 ;$ Trim load case ID $=1500$} \\
\hline \multicolumn{2}{|l|}{ DLW } & \multicolumn{4}{|c|}{$\mathrm{f}_{L M G}=1.20 ; \mathrm{f}_{L N G}=0.1477 ;$ Trim load case ID $=1800$} \\
\hline
\end{tabular}
Landing Loads

Ground Control Loads s $\checkmark$

3-point braked roll

2-point braked roll

Dynamic roll braking$$
\text { (1) }
$$

Turning 3012 (DTOW) Condition \& 4012 (DLW) Nose wheel yaw \& steering (1) Nose wheel yaw \& steering (2)

Nose wheel yaw \& steering (3)

Reversed braking

2G Taxi 3013 (DTOW)
$\& 4013$ (DLW)

3014 (DTOW) $\& 4014$ (DLW)

3015 (DTOW) $\& 4015$ (DLW) $\& 4016$ (DLW)

3017 (DTOW) $\& 4017$ (DLW)
3016 (DTOW) 


\section{Buckling and Strength Analyses}

․ Based on five analysis sets

\begin{tabular}{|c||c|c|l|}
\hline $\begin{array}{c}\text { Analysis } \\
\text { Set }\end{array}$ & $\begin{array}{c}\text { Gear } \\
\text { Configuration }\end{array}$ & $\begin{array}{c}\text { Weight } \\
\text { Condition }\end{array}$ & \multicolumn{1}{|c|}{ Load Cases } \\
\hline \hline 1 & Up & DTOW & $100,200,300,600,1100,1200,1300,1400, \& 1600$ \\
\hline 2 & Up & ZFW & $700,800,900,1000, \&$ 1700 \\
\hline 3 & Up & M2W & $400 \& 500$ \\
\hline 4 & Down & DTOW & $\begin{array}{l}3001 \sim 3017+3018 \sim 3021 \text { (emergency) + 1500 (for } \\
\text { landing) }\end{array}$ \\
\hline 5 & Down & DLW & $\begin{array}{l}4001 \sim 4017+4018 \sim 4021 \text { (emergency) + 1800 (for } \\
\text { landing) }\end{array}$ \\
\hline
\end{tabular}

a Minimum buckling load factors from each analysis set

\begin{tabular}{|c||c|c|c|c|c|c|}
\hline $\begin{array}{c}\text { Analysis } \\
\text { Set }\end{array}$ & $\begin{array}{c}\text { Gear } \\
\text { Configuration }\end{array}$ & $\begin{array}{c}\text { Weight } \\
\text { Condition }\end{array}$ & $\begin{array}{c}\text { Case } \\
\text { Number }\end{array}$ & Load Case & $\begin{array}{c}\text { Minimum } \\
\text { Buckling Load } \\
\text { Factor }\end{array}$ & Bucklin! \\
\hline \hline 1 & $\mathrm{Up}$ & DTOW & 300 & $2.5 \mathrm{G}$ pull up; M=0.48 & 0.152 & yes \\
\hline 2 & $\mathrm{Up}$ & ZFW & 1700 & $2.7 \mathrm{G}$ gust loads; $\mathrm{M}=0.89$ & 0.195 & yes \\
\hline 3 & $\mathrm{Up}$ & $\mathrm{M} 2 \mathrm{~W}$ & 400 & $2.5 \mathrm{G}$ pull up; M=2.00 & 0.151 & yes \\
\hline 4 & Down & DTOW & 3006 & Left one gear landing & 1.71 & no \\
\hline 5 & Down & DLW & 4006 & Left one gear landing & 1.52 & no \\
\hline
\end{tabular}

$\square$ Minimum margins of safety from each analysis set

\begin{tabular}{|c|c|c|c|c|c|c|}
\hline $\begin{array}{l}\text { Analysis } \\
\text { Set }\end{array}$ & $\begin{array}{c}\text { Gear } \\
\text { Configuration }\end{array}$ & $\begin{array}{c}\text { Weight } \\
\text { Condition }\end{array}$ & $\begin{array}{c}\text { Case } \\
\text { Number }\end{array}$ & Load Case & $\begin{array}{c}\text { Minimum } \\
\text { Margin of } \\
\text { Safety }\end{array}$ & Failur \\
\hline 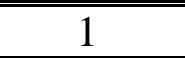 & $\overline{\mathrm{Up}}$ & DTOW & 1400 & 1.67G abrupt roll; $\mathrm{M}=0.48$ & -0.999 & \\
\hline 2 & Up & $\mathrm{ZFW}$ & 1700 & $2.7 \mathrm{G}$ gust loads; $\mathrm{M}=0.89$ & -0.998 & yes \\
\hline 3 & $\mathrm{Up}$ & M2W & 400 & $2.5 \mathrm{G}$ pull up; $\mathrm{M}=2.00$ & -0.997 & yes \\
\hline 4 & Down & DTOW & 3013 & Nose wheel yaw \& steering (1) & -0.781 & yes \\
\hline 5 & Down & DLW & 4003 & Spring back landing & -0.657 & \\
\hline
\end{tabular}

Case 300; DTOW
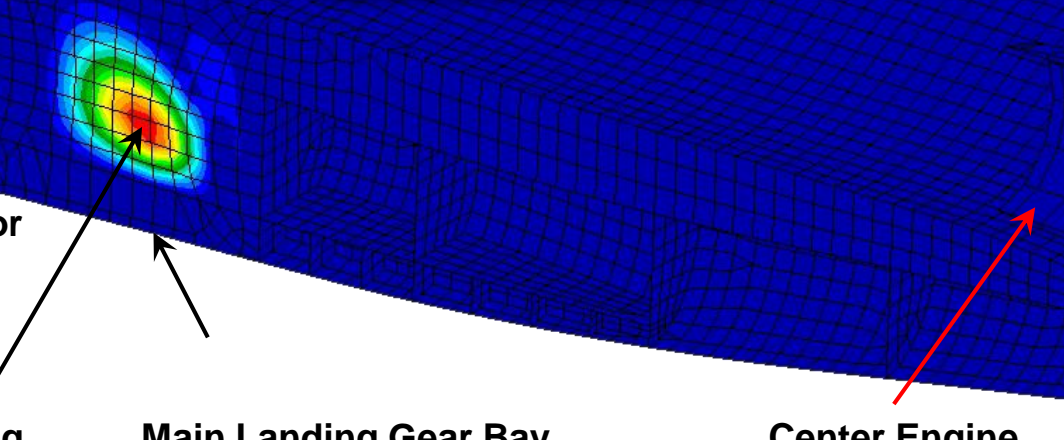

Buckling Load Factor $>1$ or

Buckling Load Factor $<0$ : requirement

Margin of safety $>0$ : requirement Safety factor $=1.5$

$$
\text { MS } \equiv \frac{\text { Failure Load }}{\text { Design Load } \times \text { Safety Factor }}-1
$$




\section{First Optimization Run}

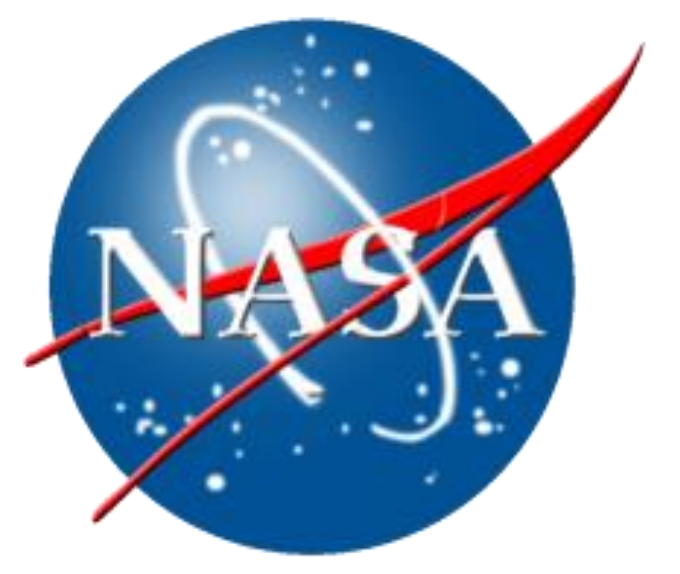


Baseline configuration is in infeasible domain. * Manually increase 111 ply thicknesses.

$>$ Wing, tail, inner-wing, and fuselage skins $\checkmark$ Three variables - $1^{\text {st }}, 3^{\text {rd }}, \& 2^{\text {nd }}=4^{\text {th }}$

$>$ Wing, inner-wing, and tail spars \& ribs and fuselage bulkheads and walls

$\checkmark$ One variable

$$
\text { - } 1^{\text {st }}=2^{\text {nd }}=3^{\text {rd }}=4^{\text {th }}
$$

$>$ Inner-wing spars \& ribs

$\checkmark$ One variable

- $5^{\text {th }}$

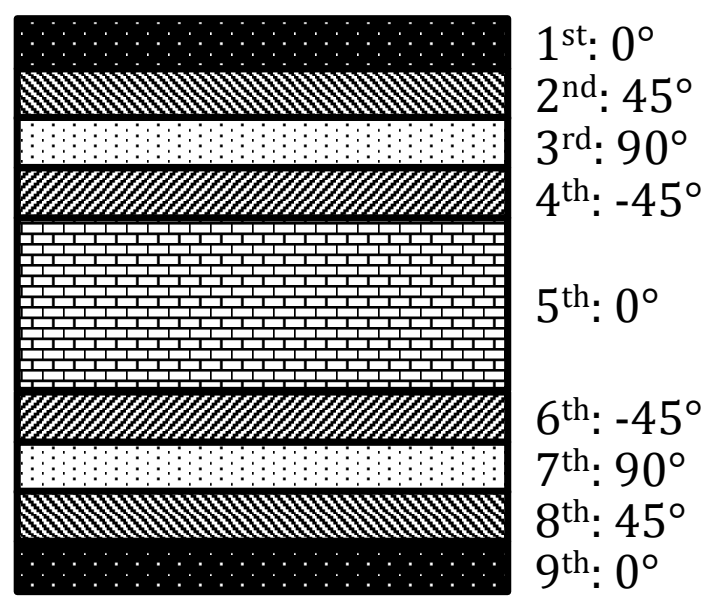

Symmetric stacking of nine plies into a composite laminate
Spars, ribs, bulkheads, \& floors

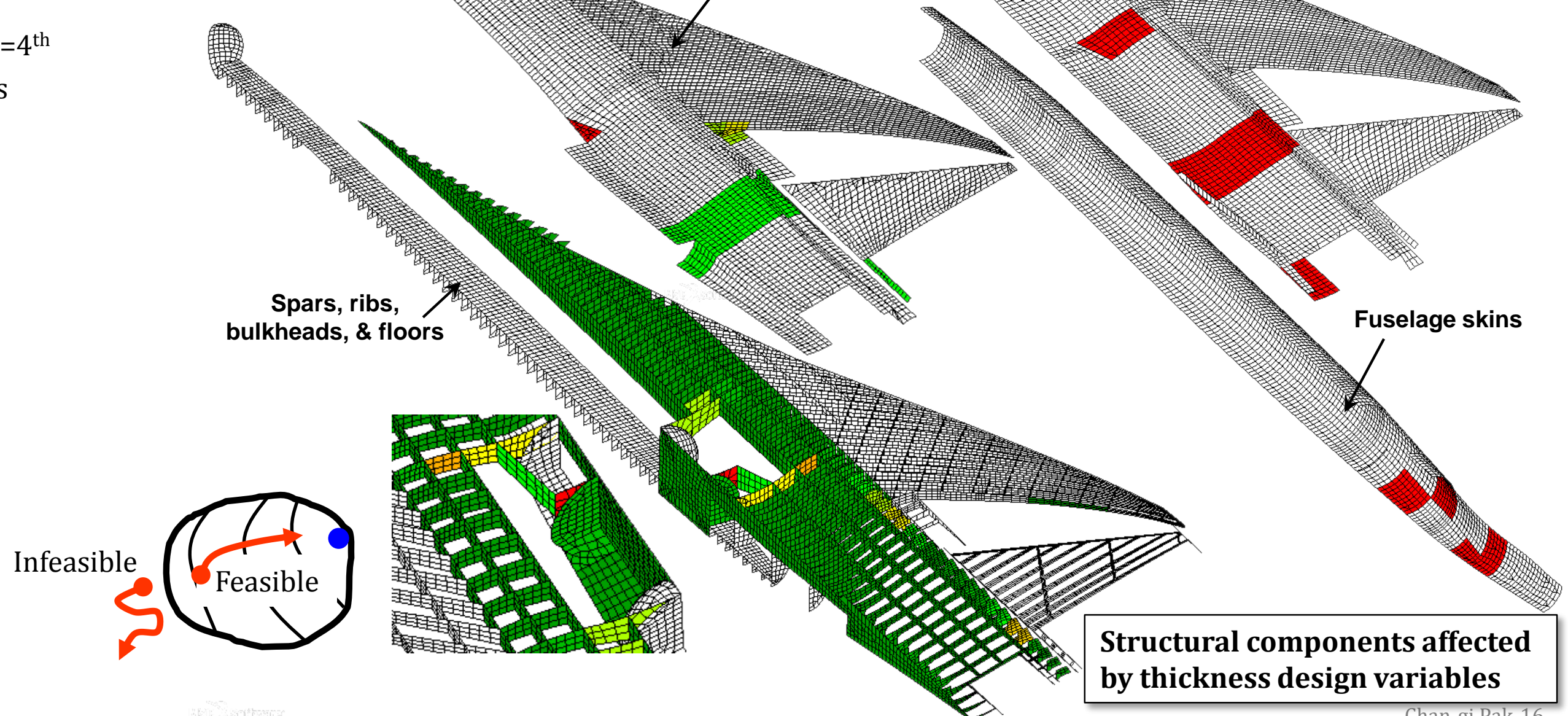

Wing tip spars \& ribs

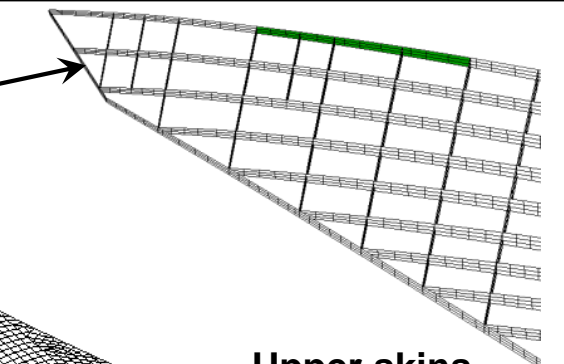

Upper skins

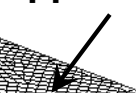




\begin{tabular}{|c||c|c|}
\hline \hline Functions & Performance indices & Notes \\
\hline \hline Objective & $F(\mathbf{X})=\left(\mathrm{PI}_{\mathrm{W}}\right)^{2}=W_{T}^{2}$ & DTOW \\
\hline $\begin{array}{c}\text { Flutter } \\
\text { constraint }\end{array}$ & $g_{j}(\mathbf{X})=\mathrm{PI}_{\mathrm{F}}=1 .-\frac{\mathrm{V}_{\mathrm{F}}}{1.15 \mathrm{~V}_{\mathrm{L}}}<0$. & $15 \%$ margin \\
$\mathrm{j}=1,2, \ldots, 6$ & Safety factor $=1.5$ \\
\hline $\begin{array}{c}\text { Buckling } \\
\text { constraint }\end{array}$ & $g_{j}(\mathbf{X})=\mathrm{PI}_{\mathrm{B}}=(1 / 2)^{2}-\{\text { positive } \min (\mathrm{BLF})-1 / 2\}^{2}<0$. \\
\hline $\begin{array}{c}\text { Strength } \\
\text { constraint }\end{array}$ & $g_{j}(\mathbf{X})=7,8, \ldots, 11$ & Safety factor $=1.5$ \\
\hline
\end{tabular}

Objective: total weight of gear up DTOW case

$\square$ Flutter constraints

* Gear Up DTOW at M=0.66, 0.89, \& 1.41

* Gear up FFEP at M=0.66, 0.89, \& 1.41

B Buckling \& strength constraints

* Minimum "buckling load factor" \& minimum "margin of safety" from five analysis sets

\begin{tabular}{|c||c|c|l|}
\hline $\begin{array}{c}\text { Analysis } \\
\text { Set }\end{array}$ & $\begin{array}{c}\text { Gear } \\
\text { Configuration }\end{array}$ & $\begin{array}{c}\text { Weight } \\
\text { Condition }\end{array}$ & \multicolumn{1}{c|}{ Load Cases } \\
\hline \hline 1 & Up & DTOW & $100,200,300,600,1100,1200,1300,1400, \& 1600$ \\
\hline 2 & Up & ZFW & $700,800,900,1000, \& 1700$ \\
\hline 3 & Up & M2W & $400 \& 500$ \\
\hline 4 & Down & DTOW & $\begin{array}{l}3001 \sim 3017+3018 \sim 3021 \text { (emergency) + 1500 (for } \\
\text { landing) }\end{array}$ \\
\hline 5 & Down & DLW & $\begin{array}{l}4001 \sim 4017+4018 \sim 4021 \text { (emergency) + 1800 (for } \\
\text { landing) }\end{array}$ \\
\hline
\end{tabular}

$O$ : Empty Fuel Empty Payload (EFEP) $\triangle$ : Full Fuel Empty Payload (FFEP)

$\diamond:$ Zero Fuel Weight (ZLW)

$\neg$ : Design Take-Off Weight (DTOW)

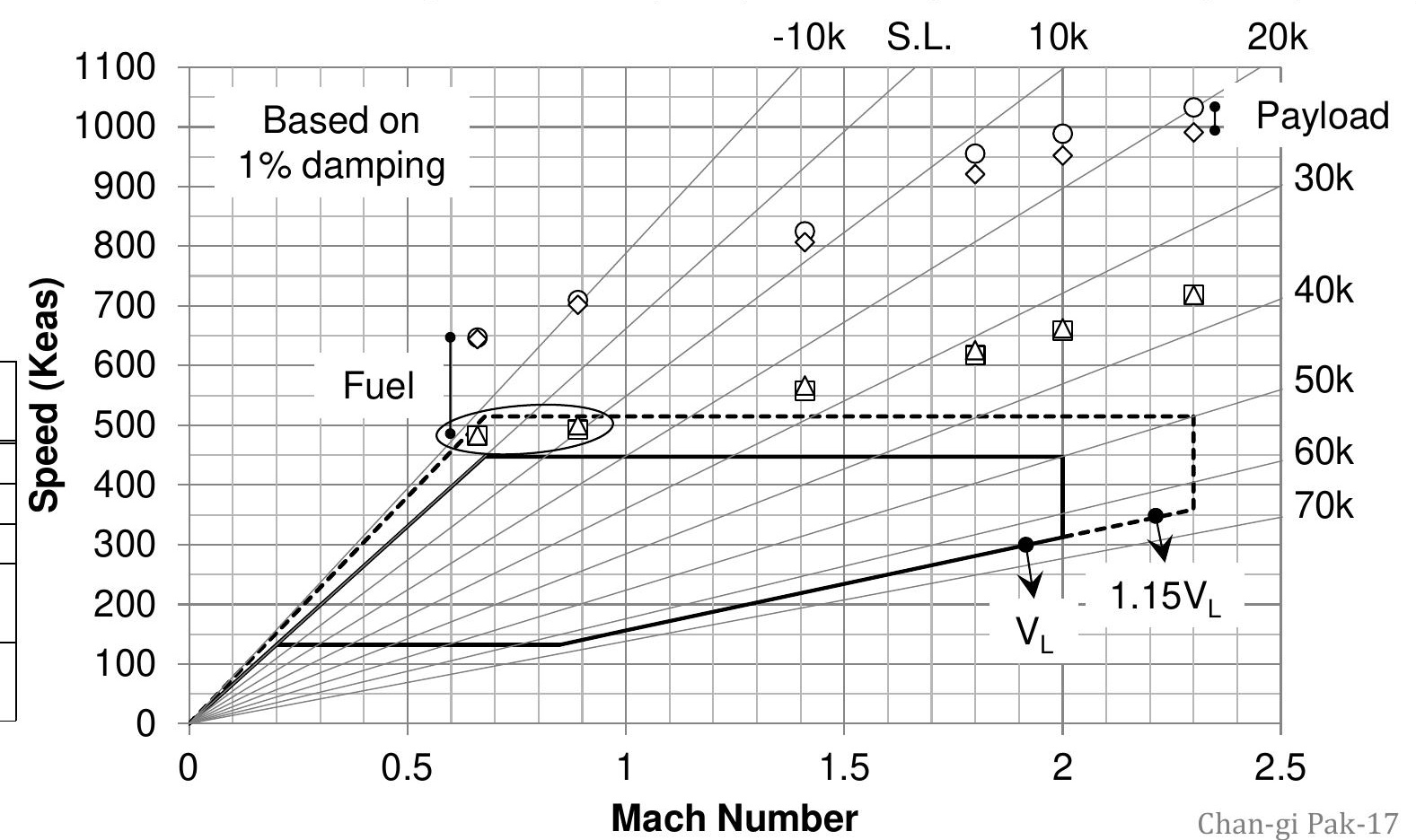




\section{First Optimization Run (continue)}

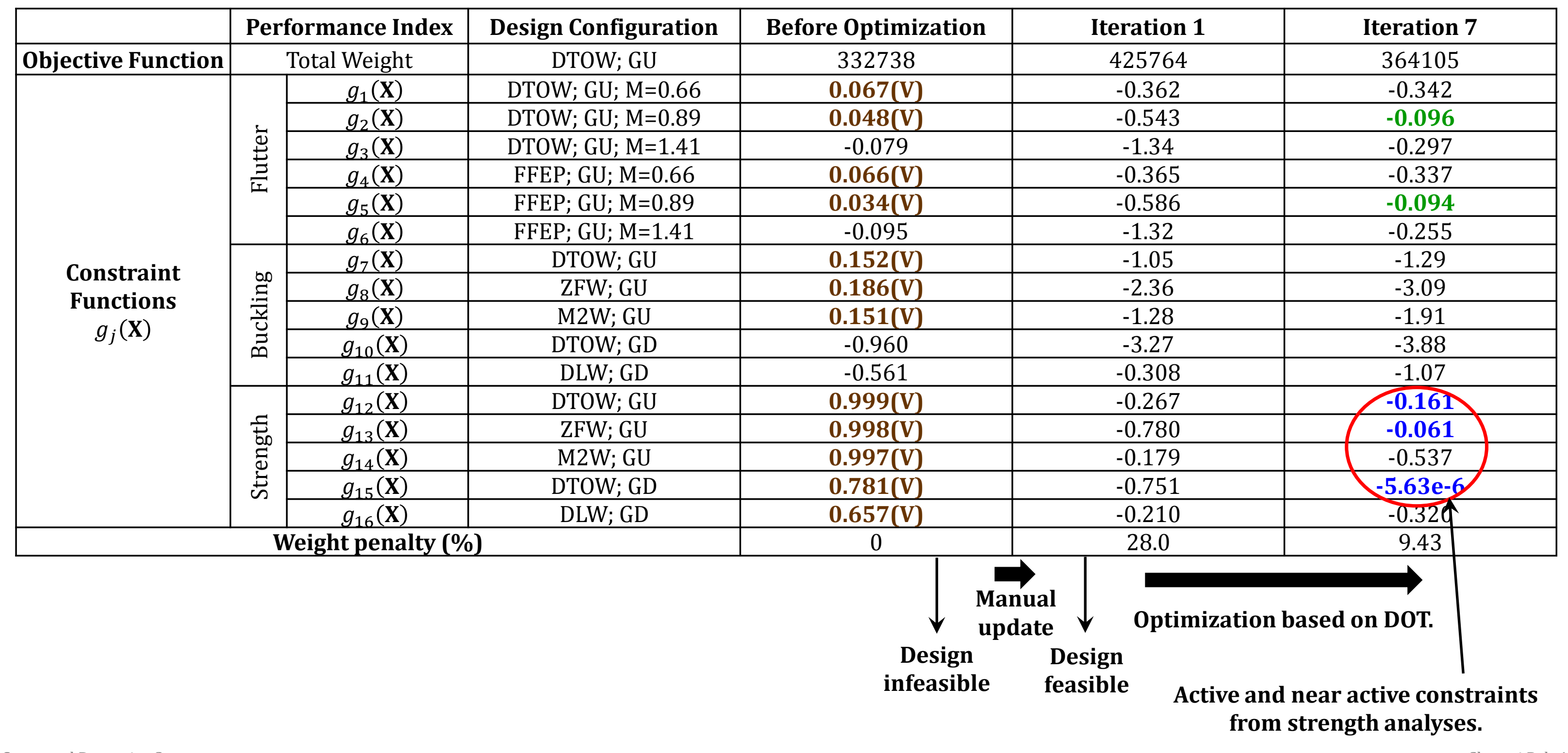




\section{First Optimization Run (continue)}

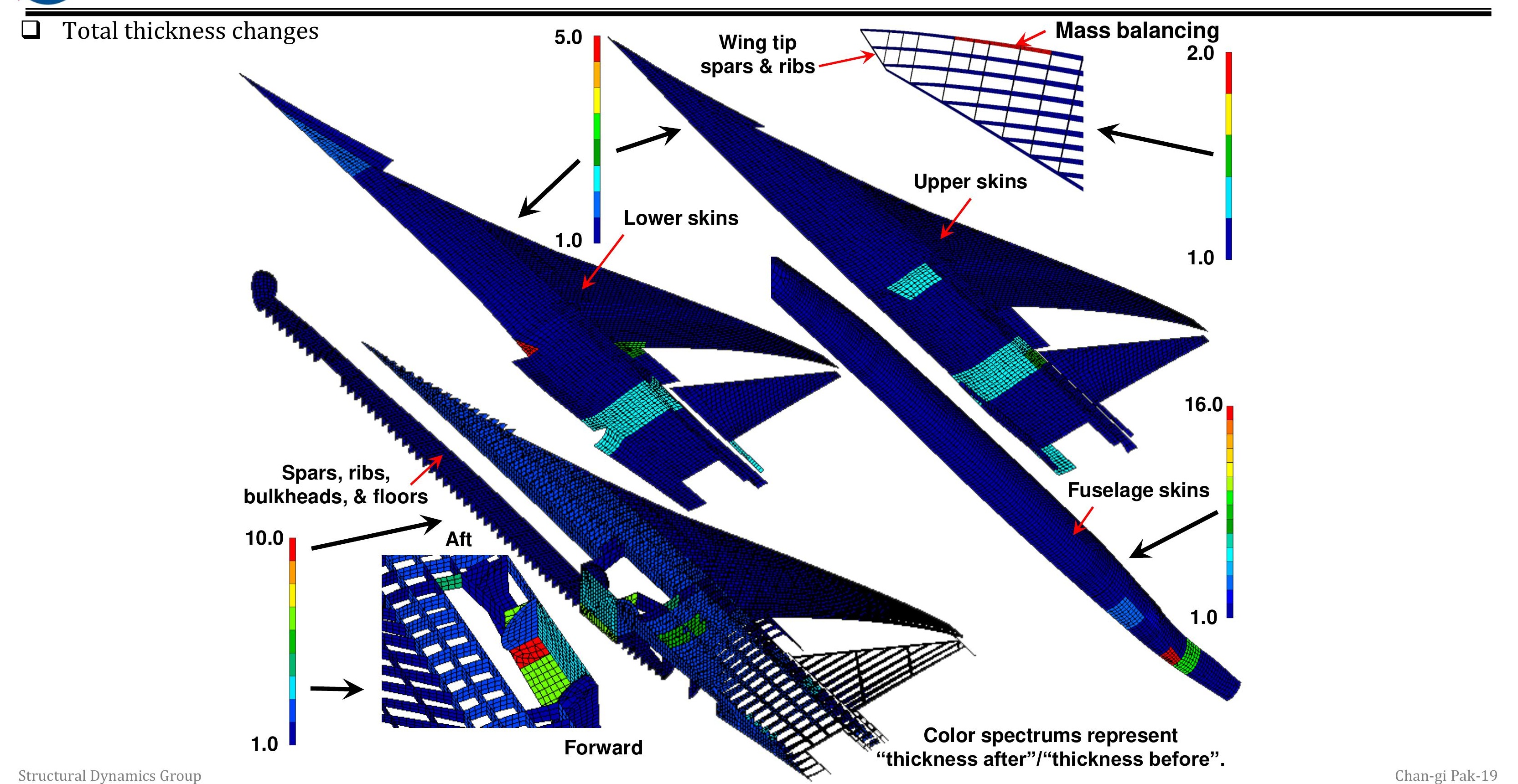


Strain distribution of the active and near active constraints

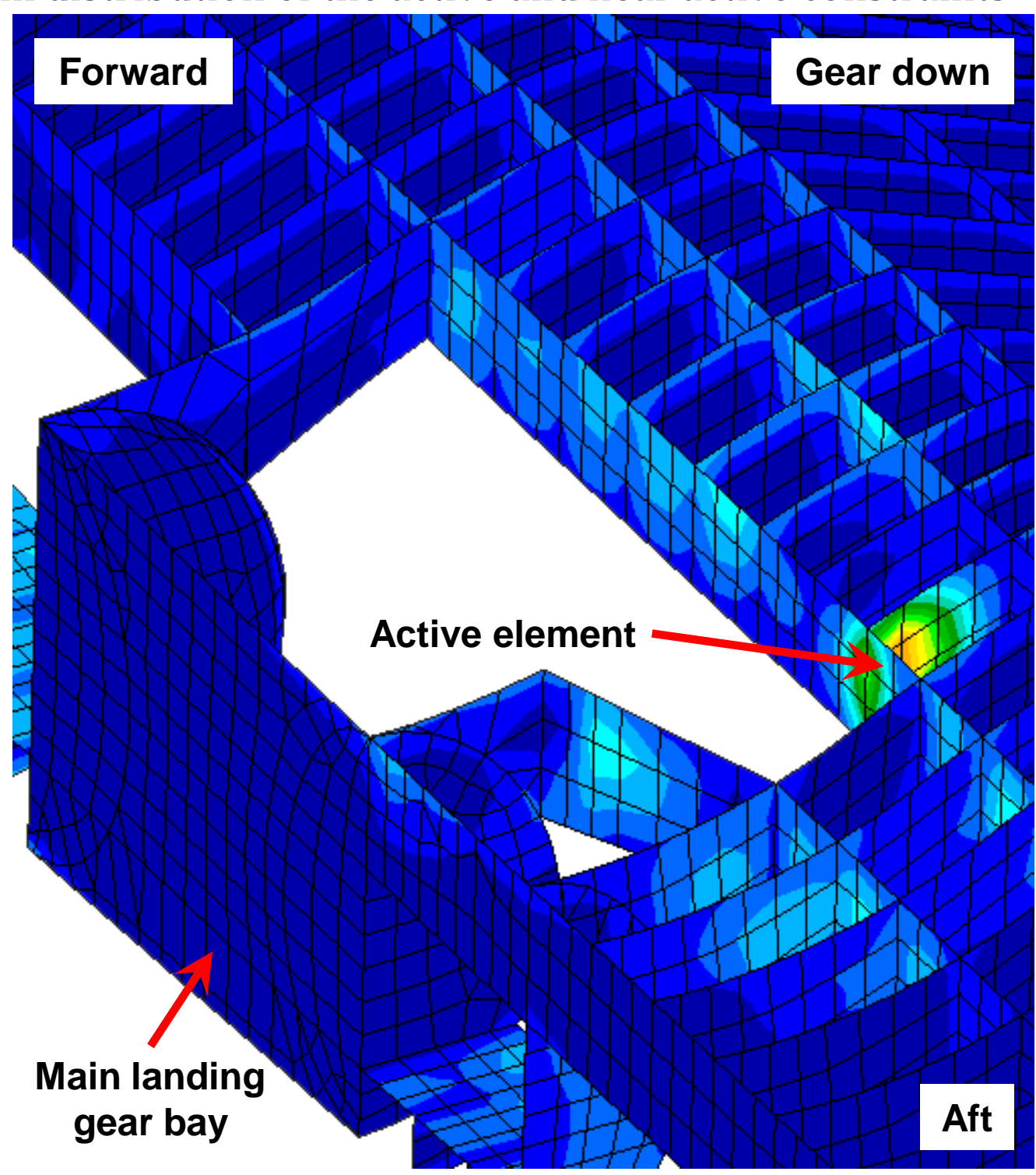

(a) Active constraint (from strength 4; load case \#3013)

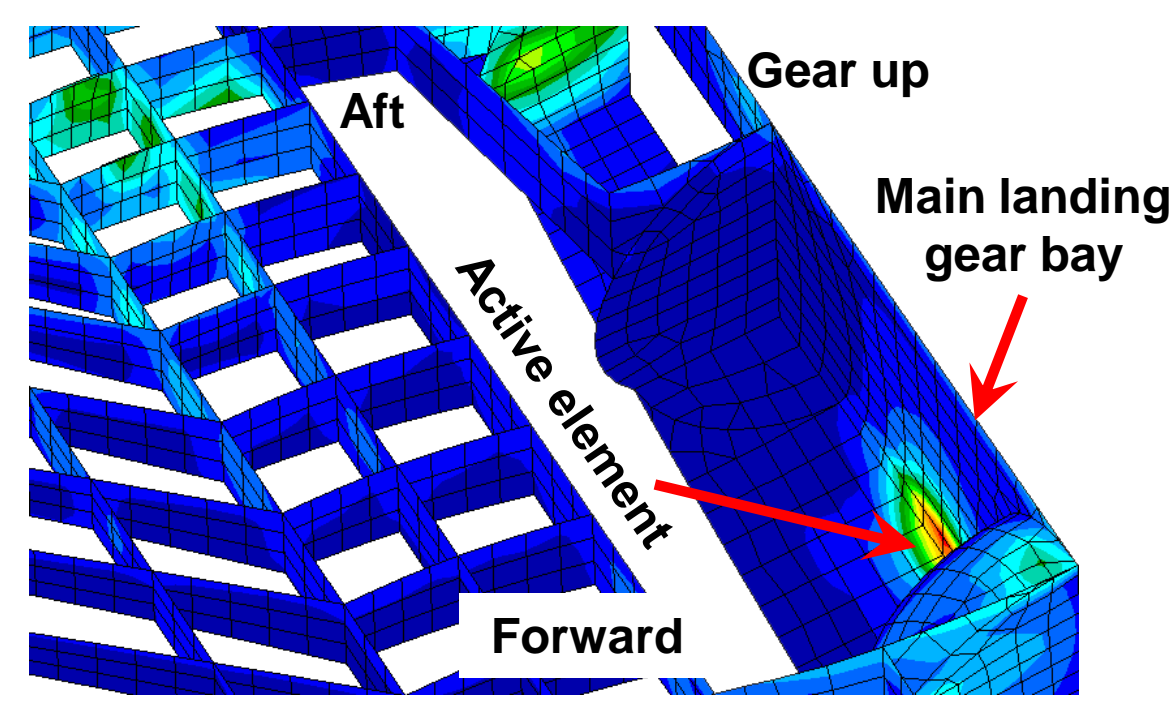

(b) Near active constraint (from strength 2; load case \#1700)

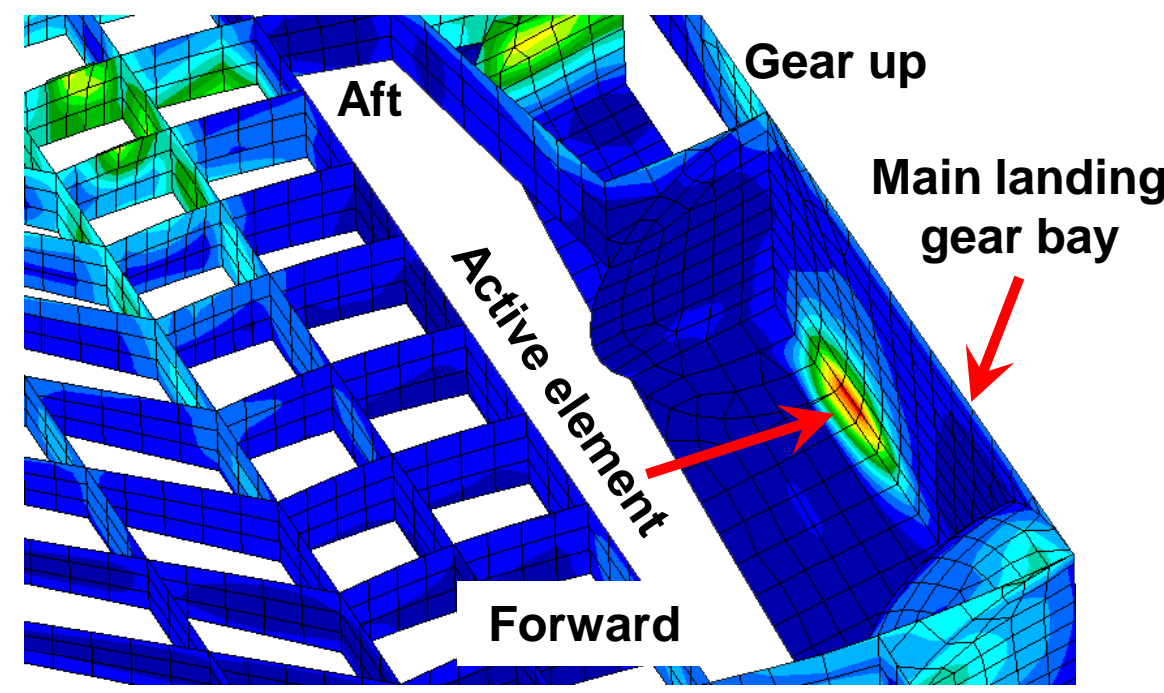

(c) Near active constraint (from strength 1; load case \#300) 


\section{Second Optimization Run}

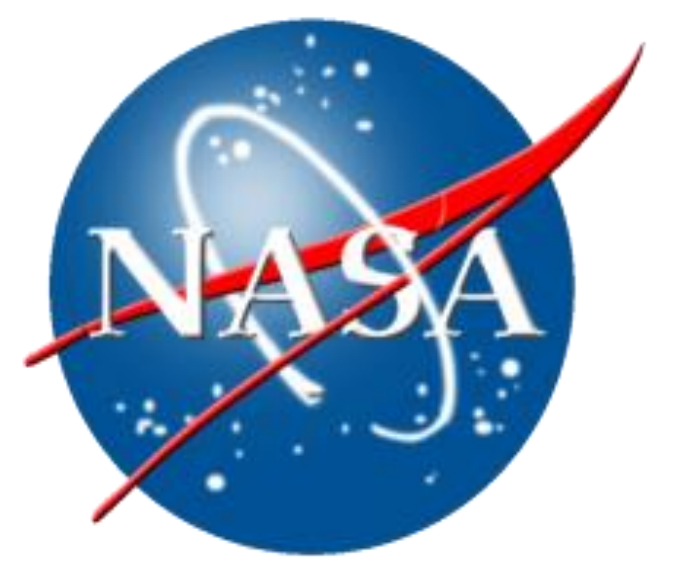




\section{Design Variables for the Second Optimization Run}

C Composite ply angles of the six zones

- Design variables are ply angles of the $2^{\text {nd }}$ and $4^{\text {th }}$ layers.

$>$ Design variable linking $2^{\text {nd }}=-4^{\text {th }}$

* Use discrete design variables

\begin{tabular}{|c|c|}
\hline Value Ranges & $\begin{array}{c}\text { Discrete } \\
\text { values }\end{array}$ \\
\hline $0.0^{\circ} \leq$ Ply angle $<2.5^{\circ}$ & $0^{\circ}$ \\
\hline $2.5^{\circ} \leq$ Ply angle $<7.5^{\circ}$ & $5^{\circ}$ \\
\hline $7.5^{\circ} \leq$ Ply angle $<12.5^{\circ}$ & $10^{\circ}$ \\
\hline $12.5^{\circ} \leq$ Ply angle $<17.5^{\circ}$ & $15^{\circ}$ \\
\hline $17.5^{\circ} \leq$ Ply angle $<22.5^{\circ}$ & $20^{\circ}$ \\
\hline $22.5^{\circ} \leq$ Ply angle $<27.5^{\circ}$ & $25^{\circ}$ \\
\hline $27.5^{\circ} \leq$ Ply angle $<32.5^{\circ}$ & $30^{\circ}$ \\
\hline $32.5^{\circ} \leq$ Ply angle $<37.5^{\circ}$ & $35^{\circ}$ \\
\hline $37.5^{\circ} \leq$ Ply angle $<42.5^{\circ}$ & $40^{\circ}$ \\
\hline $42.5^{\circ} \leq$ Ply angle $<47.5^{\circ}$ & $45^{\circ}$ \\
\hline $47.5^{\circ} \leq$ Ply angle $<52.5^{\circ}$ & $50^{\circ}$ \\
\hline $52.5^{\circ} \leq$ Ply angle $<57.5^{\circ}$ & $55^{\circ}$ \\
\hline $57.5^{\circ} \leq$ Ply angle $<62.5^{\circ}$ & $60^{\circ}$ \\
\hline $62.5^{\circ} \leq$ Ply angle $<67.5^{\circ}$ & $65^{\circ}$ \\
\hline $67.5^{\circ} \leq$ Ply angle $<72.5^{\circ}$ & $70^{\circ}$ \\
\hline $72.5^{\circ} \leq$ Ply angle $<77.5^{\circ}$ & $75^{\circ}$ \\
\hline $77.5^{\circ} \leq$ Ply angle $<82.5^{\circ}$ & $80^{\circ}$ \\
\hline $82.5^{\circ} \leq$ Ply angle $<87.5^{\circ}$ & $85^{\circ}$ \\
\hline $87.5^{\circ} \leq$ Ply angle $\leq 90.0^{\circ}$ & $90^{\circ}$ \\
\hline \multicolumn{2}{|c|}{} \\
\hline
\end{tabular}
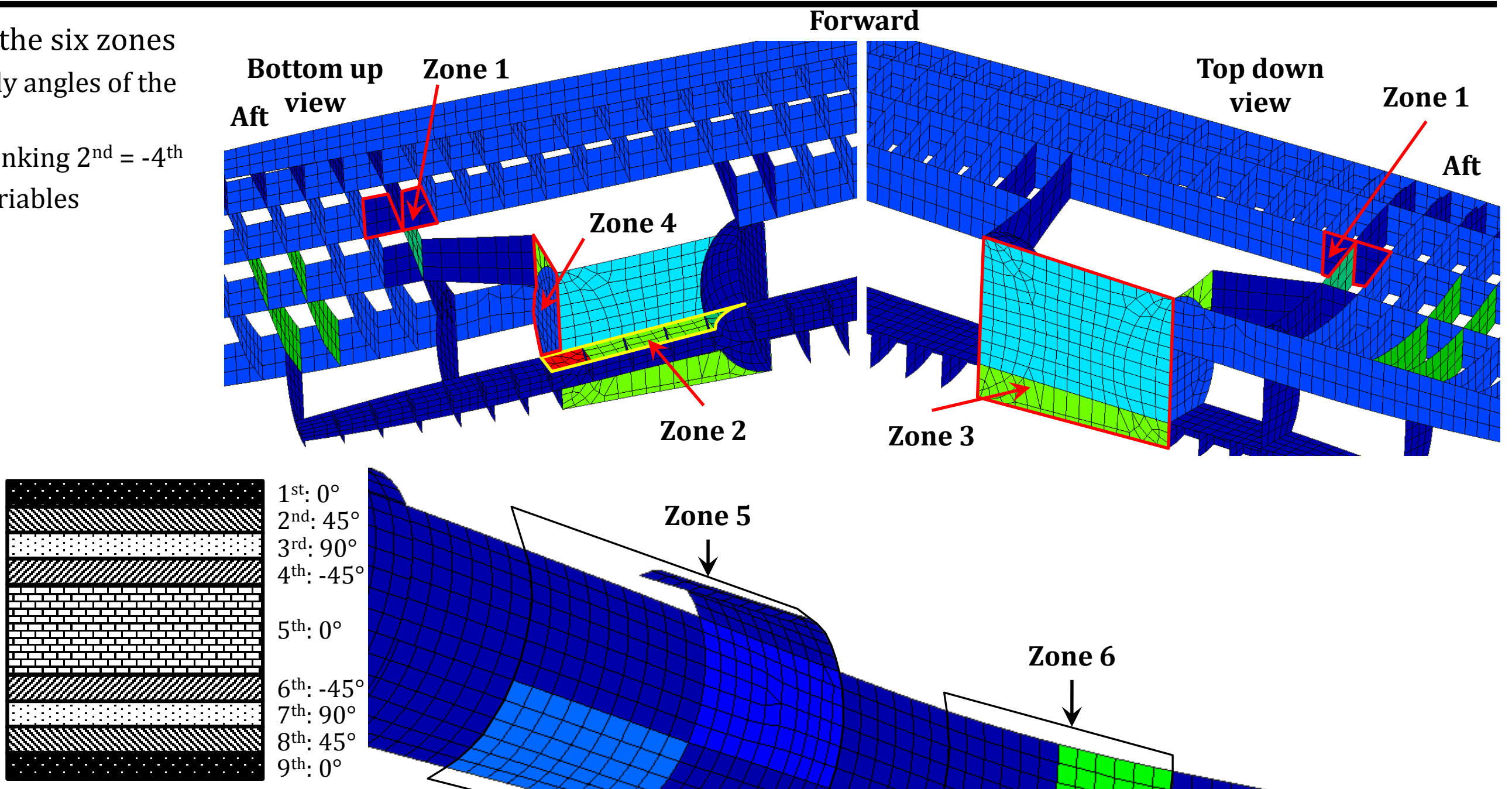

$1^{\text {st. }} 0^{\circ}$
$2^{\text {nd: }}: 45^{\circ}$
$3^{\text {rd: }} 90^{\circ}$
$4^{\text {th: }}-45^{\circ}$
$5^{\text {th }}: 0^{\circ}$
$6^{\text {th: }}-45^{\circ}$
$7^{\text {th: }}: 90^{\circ}$
$8^{\text {th }}: 45^{\circ}$
$9^{\text {th }}: 0^{\circ}$

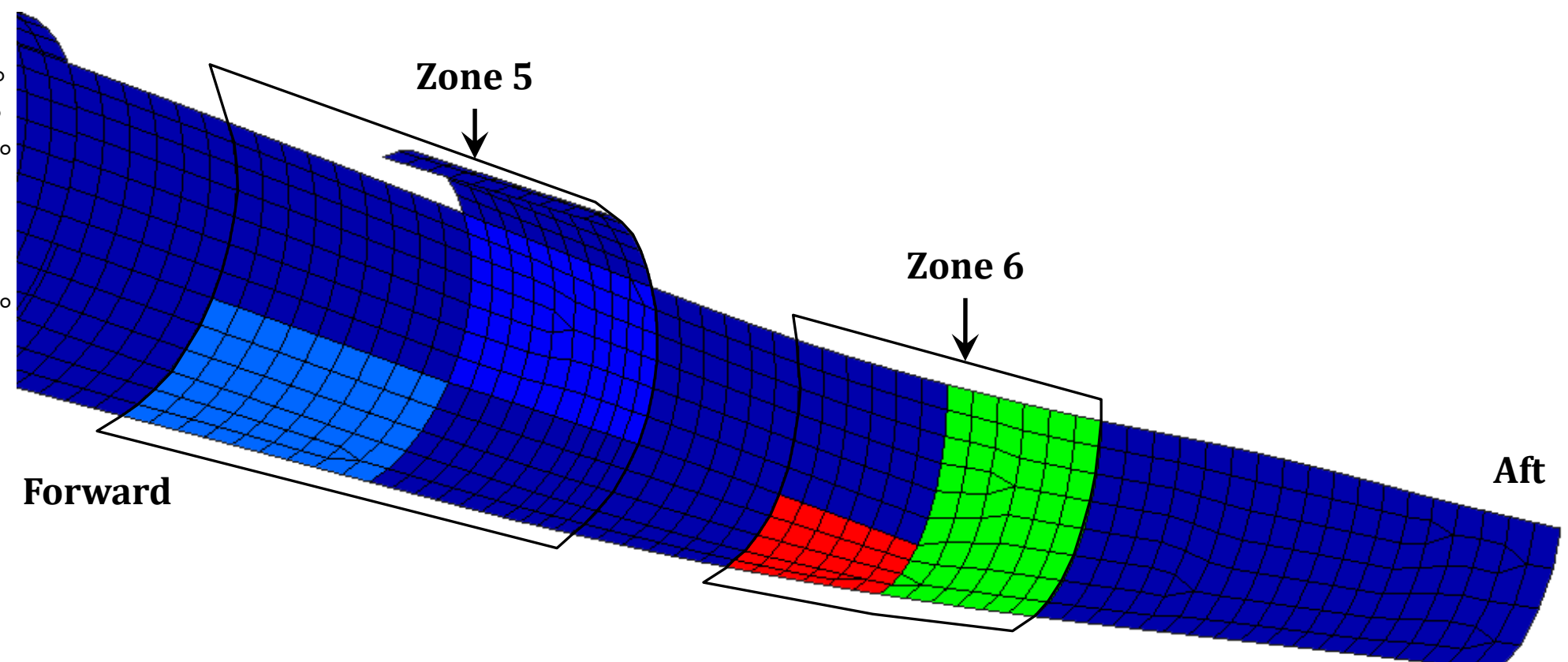




\section{Second Optimization Run}

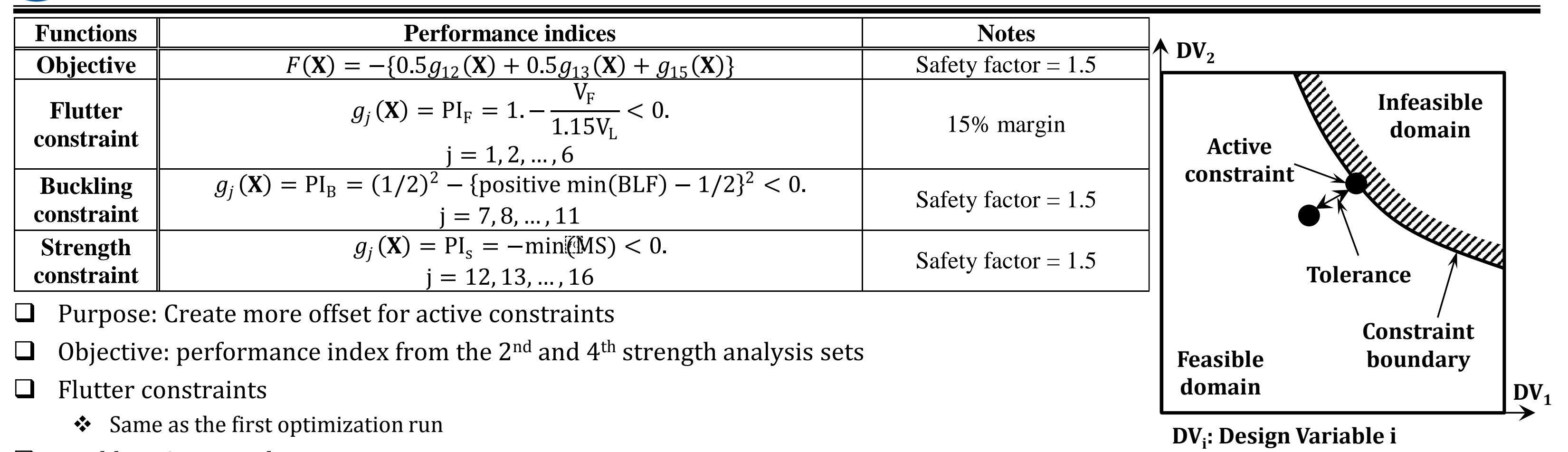

口 Buckling \& strength constraints

* Same as the first optimization run except performance indices for the objective function

\begin{tabular}{|c||c|c|l|}
\hline $\begin{array}{c}\text { Analysis } \\
\text { Set }\end{array}$ & $\begin{array}{c}\text { Gear } \\
\text { Configuration }\end{array}$ & $\begin{array}{c}\text { Weight } \\
\text { Condition }\end{array}$ & \multicolumn{1}{c|}{ Load Cases } \\
\hline \hline 1 & Up & DTOW & $100,200,300,600,1100,1200,1300,1400, \& 1600$ \\
\hline 2 & Up & ZFW & $700,800,900,1000, \& 1700$ \\
\hline 3 & Up & M2W & $400 \& 500$ \\
\hline 4 & Down & DTOW & $\begin{array}{l}3001 \sim 3017+3018 \sim 3021 \text { (emergency) + 1500 (for } \\
\text { landing) }\end{array}$ \\
\hline 5 & Down & DLW & $\begin{array}{l}4001 \sim 4017+4018 \sim 4021 \text { (emergency) + 1800 (for } \\
\text { landing) }\end{array}$ \\
\hline
\end{tabular}




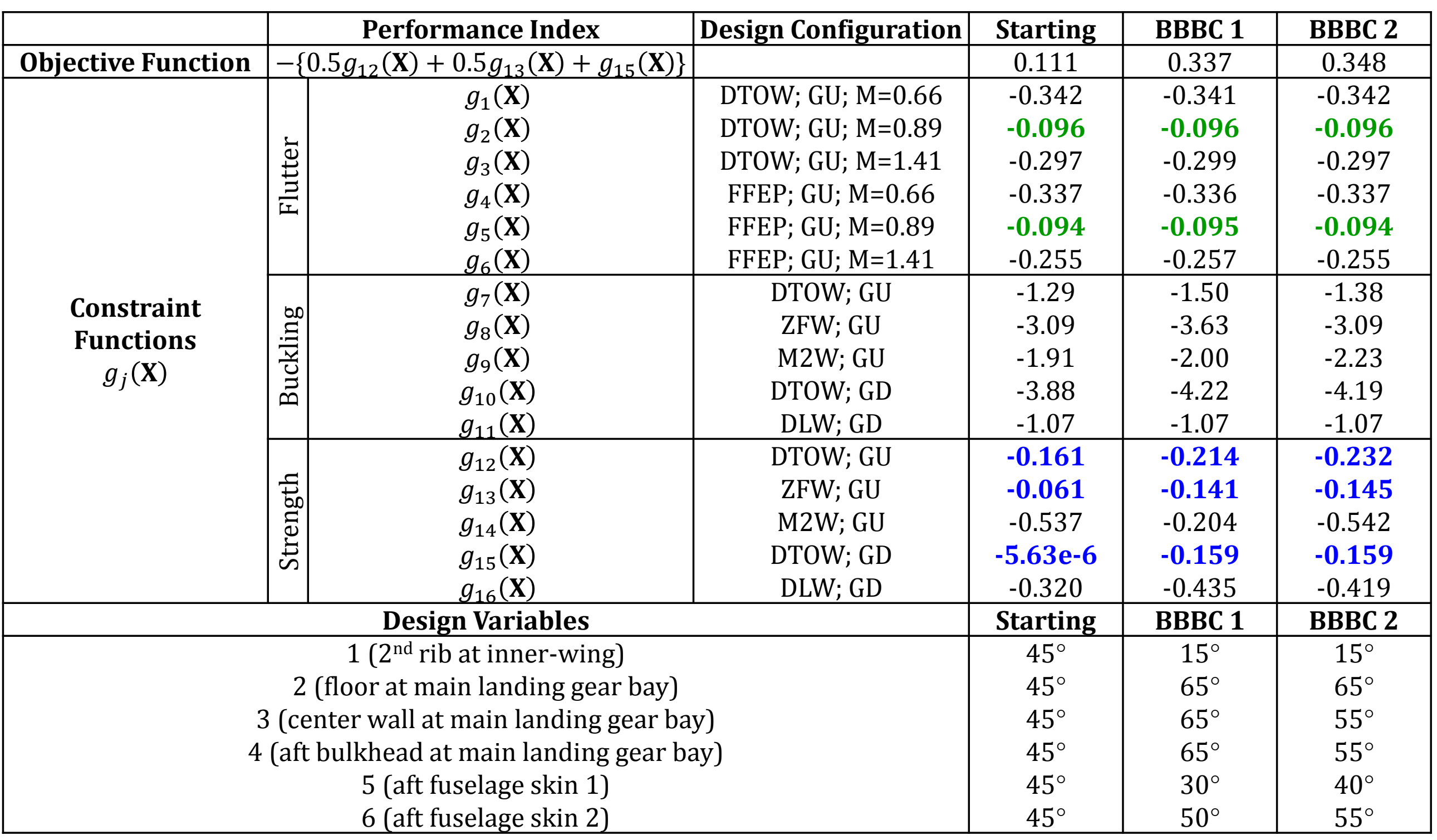

Big-Bang Big-Crunch algorithm; number of population=60; number of Big-Bang Big-Crunch=2; discrete design variables 


\section{Third Optimization Run}

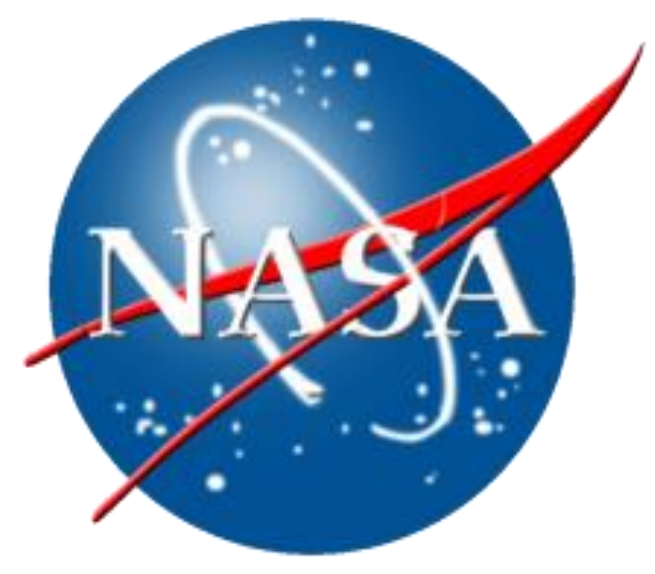




\section{Third Optimization Run}

\begin{tabular}{|c||c|c|}
\hline \hline Functions & Performance indices & Notes \\
\hline Objective & $F(\mathbf{X})=\left(\mathrm{PI}_{\mathrm{W}}\right)^{2}=W_{T}^{2}$ & DTOW \\
\hline $\begin{array}{c}\text { Flutter } \\
\text { constraint }\end{array}$ & $g_{j}(\mathbf{X})=\mathrm{PI}_{\mathrm{F}}=1 .-\frac{\mathrm{V}_{\mathrm{F}}}{1.15 \mathrm{~V}_{\mathrm{L}}}<0$. & $15 \%$ margin \\
$\mathrm{j}=1,2, \ldots, 6$ & Safety factor $=1.5$ \\
\hline $\begin{array}{c}\text { Buckling } \\
\text { constraint }\end{array}$ & $g_{j}(\mathbf{X})=\mathrm{PI}_{\mathrm{B}}=(1 / 2)^{2}-\{\text { positive } \min (\mathrm{BLF})-1 / 2\}^{2}<0$. \\
\hline $\begin{array}{c}\text { Strength } \\
\text { constraint }\end{array}$ & $g_{j}(\mathbf{X})=7,8, \ldots, 11$ & Safety factor $=1.5$ \\
\hline
\end{tabular}

$\square$ Objective: total weight of gear up DTOW case

- Flutter constraints

* Gear Up DTOW at M=0.66, 0.89, \& 1.41

* Gear up FFEP at M=0.66, 0.89, \& 1.41

B Buckling \& strength constraints

* Minimum "buckling load factor" \& minimum "margin of safety" from five analysis sets

\begin{tabular}{|c||c|c|l|}
\hline $\begin{array}{c}\text { Analysis } \\
\text { Set }\end{array}$ & $\begin{array}{c}\text { Gear } \\
\text { Configuration }\end{array}$ & $\begin{array}{c}\text { Weight } \\
\text { Condition }\end{array}$ & \multicolumn{1}{c|}{ Load Cases } \\
\hline \hline 1 & Up & DTOW & $100,200,300,600,1100,1200,1300,1400, \& 1600$ \\
\hline 2 & Up & ZFW & $700,800,900,1000, \& 1700$ \\
\hline 3 & Up & M2W & $400 \& 500$ \\
\hline 4 & Down & DTOW & $\begin{array}{l}3001 \sim 3017+3018 \sim 3021 \text { (emergency) + 1500 (for } \\
\text { landing) }\end{array}$ \\
\hline 5 & Down & DLW & $\begin{array}{l}4001 \sim 4017+4018 \sim 4021 \text { (emergency) + 1800 (for } \\
\text { landing) }\end{array}$ \\
\hline
\end{tabular}




\section{Third Optimization Run}

\begin{tabular}{|c|c|c|c|c|c|c|c|c|c|c|c|}
\hline & \multicolumn{2}{|c|}{$\begin{array}{c}\begin{array}{c}\text { Performance } \\
\text { Index }\end{array} \\
\end{array}$} & $\begin{array}{c}\text { Design } \\
\text { Configuration } \\
\end{array}$ & Starting & Iteration 1 & Iteration 2 & Iteration 3 & Iteration 4 & Iteration 5 & Iteration 6 & Iteration 7 \\
\hline \begin{tabular}{|l|} 
Objective \\
Function
\end{tabular} & \multicolumn{2}{|c|}{ Total Weight } & DTOW; GU & 364105 & 363810 & & & & & & \\
\hline \multirow{13}{*}{$\begin{array}{c}\text { Constraint } \\
\text { Functions } \\
g_{j}(\mathbf{X})\end{array}$} & \multirow{5}{*}{$\sum_{\frac{ \pm}{ \pm}}^{\mathscr{D}}$} & $g_{1}(\mathbf{X})$ & DTOW; M=0.66 & -0.342 & -0.341 & & & & & & \\
\hline & & $g_{2}(\mathbf{X})$ & DTOW; $M=0.89$ & -0.096 & -0.096 & & & & & & \\
\hline & & $g_{3}(\mathbf{X})$ & DTOW; $M=1.41$ & -0.297 & -0.297 & & & & & & \\
\hline & & $g_{4}(\mathbf{X})$ & FFEP; $M=0.66$ & -0.337 & -0.336 & & & & & & \\
\hline & & $g_{5}(\mathbf{X})$ & FFEP; $M=0.89$ & -0.094 & -0.094 & & & & & & \\
\hline & \multirow{5}{*}{ 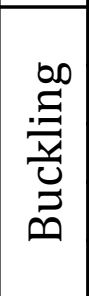 } & $g_{7}(\mathbf{X})$ & DTOW; GU & -1.38 & -1.38 & & & & & & \\
\hline & & $g_{8}(\mathbf{X})$ & ZFW; GU & -3.09 & -3.44 & & & & & & \\
\hline & & $g_{9}(\mathbf{X})$ & M2W; GU & -2.23 & -2.04 & & & & & & \\
\hline & & $g_{10}(\mathbf{X})$ & DTOW; GD & -4.19 & -3.95 & & & & & & \\
\hline & & $g_{11}(\mathbf{X})$ & DLW; GD & -1.07 & -1.07 & & & & & & \\
\hline & \multirow{3}{*}{ 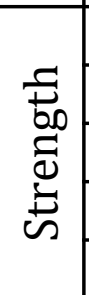 } & $g_{12}(\mathbf{X})$ & DTOW; GU & -0.232 & -0.227 & & & & & & \\
\hline & & $g_{13}(\mathbf{X})$ & ZFW; GU & -0.145 & -0.141 & & & & & & \\
\hline & & $g_{14}(\mathbf{X})$ & M2W; GU & -0.542 & -0.261 & & & & & & \\
\hline \multicolumn{4}{|c|}{ Weight penalty (\%) } & 9.43 & 9.34 & & & & & & \\
\hline
\end{tabular}

Optimization based on DOT. 


\section{Conclusions}

The Lockheed Martin's pre-matured N+2 LSCT aircraft is optimized in this study through the use of a multidisciplinary design optimization tool developed at the NASA AFRC.

- The baseline design of the pre-matured N+2 LSCT aircraft was infeasible when ZAERO based aeroelastic analyses were used.

* This probably means that the aerodynamic loads distribution computed using ZAERO trim analysis are different than the MSC Nastran generated aerodynamic loads.

The starting configuration of the optimization run should be an achievable design and weight penalty for this was $93,026 \mathrm{lb}$.

* $\mathbf{2 8 . 0} \%$ increase from baseline

During the first optimization run, the weight reduction was $61,659 \mathrm{lb}$, and therefore weight penalty at the end of the first optimization run is $31,367 \mathrm{lb}$.

* Optimization was based on DOT optimizer

* Active constraint: minimum margin of safety value is associated with the structural component located at the second rib of the inner wing near the main landing gear bay area.

$>$ Nose wheel yaw and steering case number 1

* First near active constraint: minimum margin of safety value at the floor of main landing gear bay

$>2.7 \mathrm{~g}$ gust load case at Mach 0.89 and altitude of $20,000 \mathrm{ft}$

- 9.4\% increase from baseline

* Second near active constraints: flutter speeds with DTOW and FFEP at Mach 0.89

$>$ Mass balancing effect to increase the flutter speeds

The second optimization run was prepared to increase tolerance distance for the active and the first near active constraints.

* Create more room for reducing total weight of the aircraft

* Use six ply angles as design variables

* Optimization was based on Big-Bang Big-Crunch algorithm with discrete design variables.

* Can't change weight property, but can change strength property. Therefore, can create tolerance for future weight optimization run 


\section{Questions?}

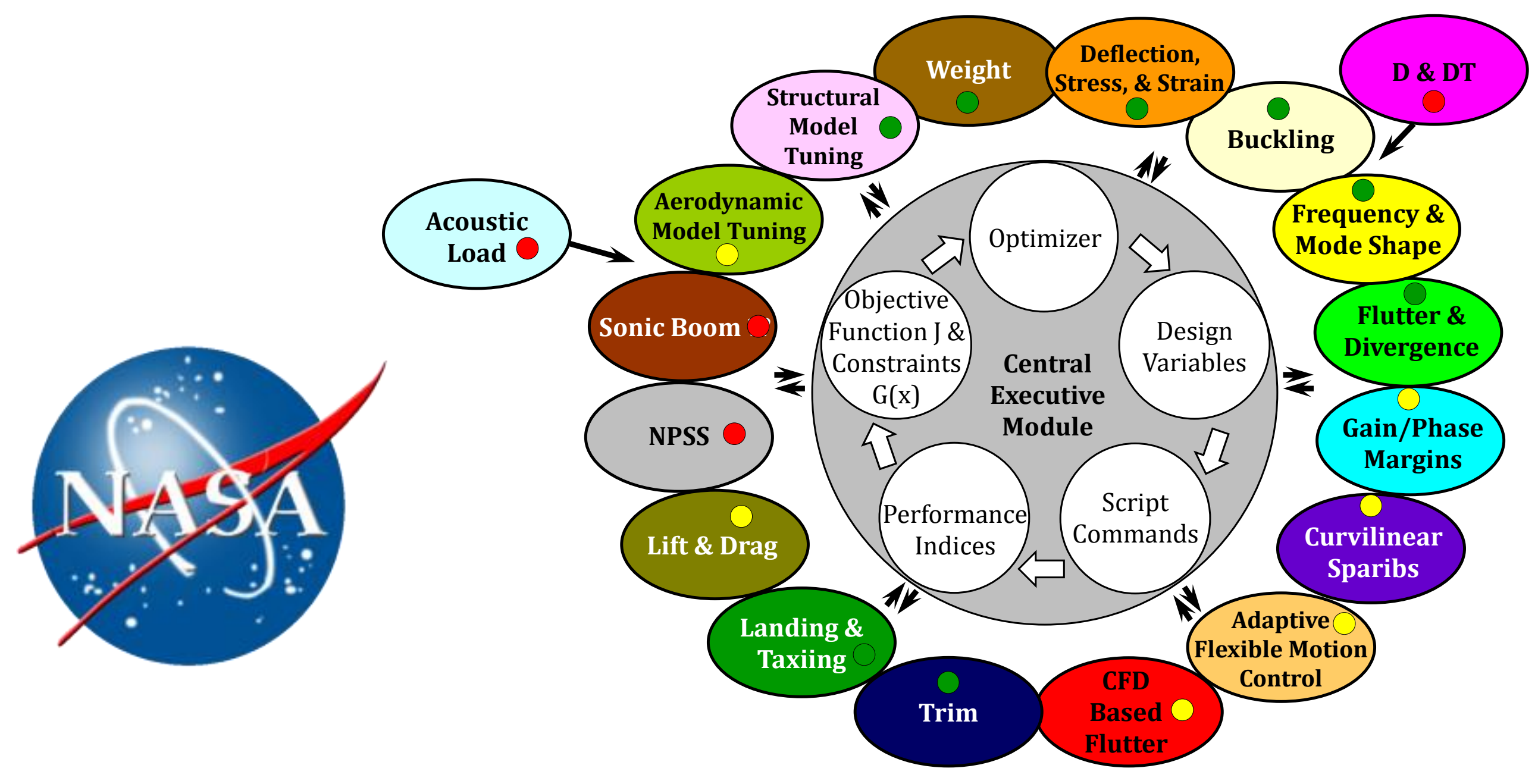


$\square \quad$ Use lifting surface based aerodynamics

* Use curvilinear sparibs to further reduce the weight of N+2 LSCT

$>\quad$ Find \% weight reduction through curvilinear sparibs technique

* Add active control design variables

$>$ Use aeroelastic tailoring up to $\mathrm{V}_{\mathrm{L}}$

$>$ Use active control between $\mathrm{V}_{\mathrm{L}}$ and $1.15 \mathrm{~V}_{\mathrm{L}}$

$>\quad$ Find \% weight reduction through game changing approach

$\square \quad$ Use CFD based aerodynamics

* Use more accurate air loads for optimizations 


\section{Backup: Object-Oriented MDO Tool}

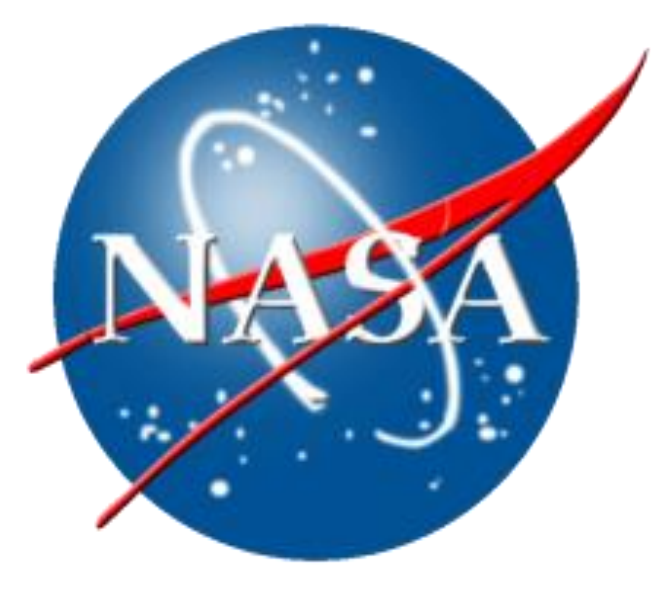




\section{Object-Oriented MDO tool}

- Optimization is based on in-house Object-Oriented Optimization tool

* Equivalent to the following codes

$>$ Open MDAO, Model Center, Visual Doc, etc.

* Four optimizer codes are available.

$>$ Gradient based algorithms (Local optimizers)

$\checkmark$ DOT

$\checkmark$ ADS

$>$ Global optimizers (Gradient free algorithms)

$\checkmark$ Genetic Algorithm

$\checkmark$ Big-Bang Big-Crunch Algorithm

U Update design pre-processor module

* Update MSC/NASTRAN input file

口 Modal analysis module

* Perform modal analysis using MSC/NASTRAN sol. 103

* Save following data

$>$ Total weight, CG location, mass moment of inertia

$>$ Frequencies \& mode shapes and global mass matrix

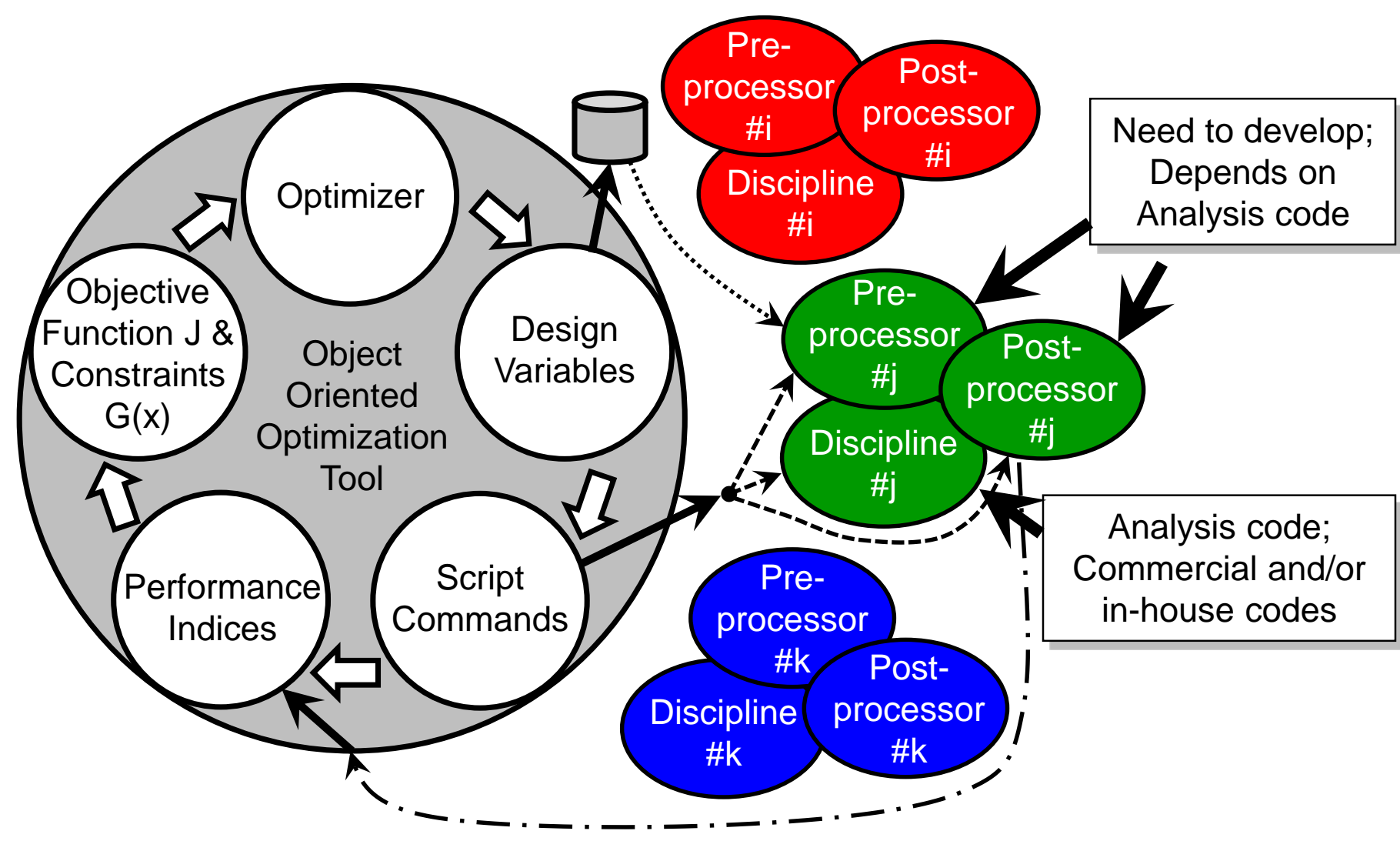

Weight post-processor module

* Use MSC/NASTRAN sol 103 results for small weight.

$>$ MSC/NASTRAN results has number of digit issue.

* Use in-house weight computation code for large weight.

$$
\mathrm{PI}_{\mathrm{W}}=W_{T}
$$


Flutter analysis and flutter post-processor modules

* Use ZAERO code for flutter analyses

* Use an in-house flutter speed tracking program

$$
\mathrm{V}_{\mathrm{F}}>1.15 \mathrm{~V}_{\mathrm{L}} \quad 1 .-\frac{\mathrm{V}_{\mathrm{F}}}{1.15 \mathrm{~V}_{\mathrm{L}}}<0 . \quad \mathrm{PI}_{\mathrm{F}} \equiv 1 .-\frac{\mathrm{V}_{\mathrm{F}}}{1.15 \mathrm{~V}_{\mathrm{L}}}
$$

Update ZAERO pre-processor, trim analysis, trim loads pre-processor modules * Update ZAERO input data

$>$ Based on total weight, CG locations, moment of inertias, and global mass matrix

* Use ZAERO code for trim analysis

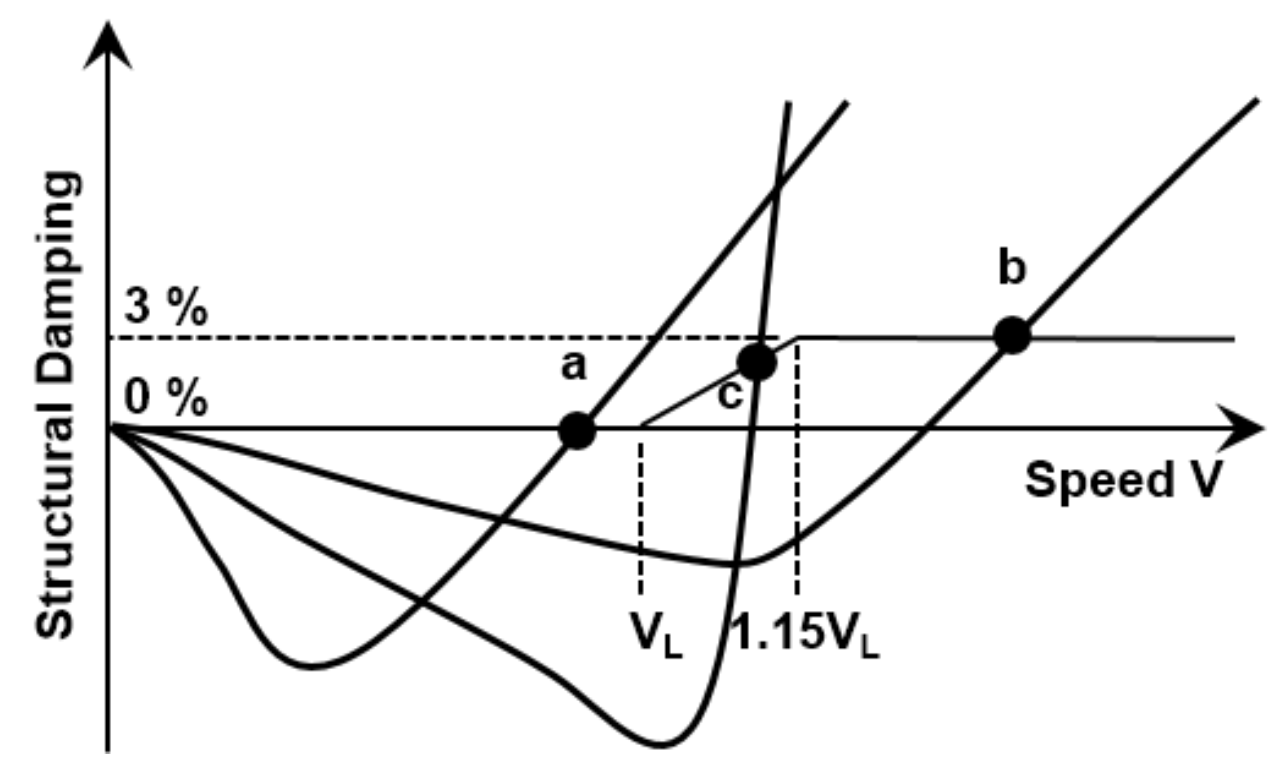

$>$ Create design loads for various design configurations

- Post-process the splined loads

$>$ Create symmetric and anti-symmetric loads

ㄴ Landing and ground control loads pre-processor module

* Compute corresponding design loads using in-house code

$>$ Landing loads

$>$ Ground control loads

$>$ Emergency landing loads 
Buckling and strength analyses and strength post-processor modules

* Based on MSC/NASTRAN sol. 105

* Use in-house strength post-processor code

* Safety factor of 1.5 is used for all metal and composite materials in this study.

Design Load $\times$ Safety Factor $<$ Failure Load $\quad 1-\frac{\text { Failure Load }}{\text { Design Load } \times \text { Safety Factor }}<0$

$\mathrm{MS} \equiv \frac{\text { Failure Load }}{\text { Design Load } \times \text { Safety Factor }}-1 . \quad \mathrm{PI}_{\mathrm{S}} \equiv-\min (\mathrm{MS})$

B Buckling post-processor module

* Use in-house code

* Buckling Load Factor (BLF) $0 \leq \mathrm{BLF} \leq 1$ : Buckling predicted $\quad \mathrm{BLF}<0$ or $\mathrm{BLF}>1$ : Buckling not predicted

$>$ Buckling predicted: $0 \leq \mathrm{BLF} \leq 1 \Rightarrow-1 / 2 \leq \mathrm{BLF}-1 / 2 \leq 1 / 2 \Rightarrow(\mathrm{BLF}-1 / 2)^{2} \leq(1 / 2)^{2}$

Buckling not predicted: $(\mathrm{BLF}-1 / 2)^{2}>(1 / 2)^{2} \longrightarrow(1 / 2)^{2}-\left(\mathrm{BLF}-\frac{1}{2}\right)^{2}<0$.

$$
\mathrm{PI}_{\mathrm{B}} \equiv(1 / 2)^{2}-\{\text { positive } \min (\mathrm{BLF})-1 / 2\}^{2}
$$

